\title{
Bifurcation in a Discrete-Time Piecewise Constant Dynamical System
}

\author{
Chenmin Hou ${ }^{1}$ and Sui Sun Cheng $^{2}$ \\ ${ }^{1}$ Department of Mathematics, Yanbian University, Yanji 133002, China \\ ${ }^{2}$ Department of Mathematics, Tsing Hua University, Hsinchu, Taiwan 30043, China
}

Correspondence should be addressed to Sui Sun Cheng; sscheng@math.nthu.edu.tw

Received 15 August 2012; Revised 27 February 2013; Accepted 28 February 2013

Academic Editor: Raghib Abu-Saris

Copyright (c) 2013 C. Hou and S. S. Cheng. This is an open access article distributed under the Creative Commons Attribution License, which permits unrestricted use, distribution, and reproduction in any medium, provided the original work is properly cited.

The study of recurrent neural networks with piecewise constant transition or control functions has attracted much attention recently because they can be used to simulate many physical phenomena. A recurrent and discontinuous two-state dynamical system involving a nonnegative bifurcation parameter is studied. By elementary but novel arguments, we are able to give a complete analysis on its asymptotic behavior when the parameter varies from 0 to $\infty$. It is hoped that our analysis will provide motivation for further results on large-scale recurrent $\mathrm{McCulloch-Pitts-type} \mathrm{neural} \mathrm{networks} \mathrm{and} \mathrm{piecewise} \mathrm{continuous} \mathrm{discrete-time} \mathrm{dynamical} \mathrm{systems.}$

\section{Introduction}

It is generally accepted that the McCulloch-Pitts model of a neural network can be used as the components of computerlike systems. That is, where neural networks are commonly used to learn something, a McCulloch-Pitts neuron is constructed to do a particular job. Although the same job can be done by means of traditional Boolean components, it is interesting to see how it all works using components which are closer to "biological" components. One particular important component can be described by means of the step (activation) function defined by

$$
f_{\sigma}(u)= \begin{cases}1 & \text { if } u \leq \sigma \\ -1 & \text { if } u>\sigma\end{cases}
$$

with a nonnegative (threshold) real parameter $\sigma$. Roughly, a neuron may receive an inhibitory (indicated by a -1 sign) value if the input signal has strength exceeding the biological threshold $\sigma$ and otherwise it remains intact with an excitory (indicated by $a+1$ sign) value.

Therefore, if we let $x_{n}$ be the state value of a neural unit during the time period $n$, then the following recurrence relation:

$$
x_{n}=F\left(f_{\sigma}\left(x_{n-1}\right), x_{n-2}\right)
$$

may be used to describe a one-neuron McCulloch-Pitts system where the state value is updated from the two most recent state values. There are now many studies that are concerned with such neural networks.

For a neural network system that contains two or more neural units, things are much more complicated, and a variety of models can be designed. In usual practice, we can build a mathematical model and use it to generate simulation results, and from these results, we may understand the general properties of our models. What is now more difficult but important is to make mathematical conclusions that may provide a full understanding of all the properties observed.

In this paper, we consider a two-neuron dynamical neural network system:

$$
\begin{aligned}
& x_{n}=a x_{n-2}+(1-a) f_{\sigma}\left(y_{n-1}\right), \\
& y_{n}=a y_{n-2}+(1-a) f_{\sigma}\left(x_{n-1}\right)
\end{aligned}
$$

for $n \in \mathbf{N}=\{0,1,2, \ldots\}$, where $a \in(0,1)$, and $f_{\sigma}: \mathbf{R} \rightarrow \mathbf{R}$ is the function in. Note that if we make the change of variables

$$
x_{n}=\frac{1-a}{b} u_{n}, \quad y_{n}=\frac{1-a}{b} v_{n}
$$


where $b$ is an arbitrary positive number, then (3) becomes the "more general" system:

$$
\begin{aligned}
& u_{n}=a u_{n-2}+b f_{b \sigma /(1-a)}\left(v_{n}\right) \\
& v_{n}=a v_{n-2}+b f_{b \sigma /(1-a)}\left(u_{n}\right),
\end{aligned}
$$

and hence all properties of (5) can be inferred from those of (3).

In this paper, we are concerned with the asymptotic behavior of (3) when the nonnegative parameter $\sigma$ is treated as a bifurcation variable and allowed to vary from 0 to $\infty$ (cf. $[1,2])$. What is interesting is that a complete asymptotic and bifurcation analysis can be obtained. With such success, it is expected that more general discontinuous recurrent McCulloch-Pitts-type neural networks [3] can be dealt with to some extent in a similar manner.

To simplify matters, note that if we let $z=(x, y)$ and

$$
F_{\sigma}(z)=\left(f_{\sigma}(y), f_{\sigma}(x)\right),
$$

then the above system (3) can be written as

$$
z_{n}=a z_{n-2}+a^{\prime} F_{\sigma}\left(z_{n-1}\right), \quad n \in \mathbf{N}
$$

where we write $z_{n}=\left(x_{n}, y_{n}\right)$ and $a^{\prime}=1-a$ for the sake of convenience. The above vector equation is a three-term recurrence relation. Hence for given $z_{-2}$ and $z_{-1}$ in the plane, a unique sequence $\left\{z_{k}\right\}_{k=-2}^{\infty}$ can be calculated from it. Such a sequence is called a solution of (7) determined by $z_{-2}$ and $z_{-1}$.

Depending on the locations of $z_{-2}$ and $z_{-1}$, it is clear that the behavior of the corresponding solution may differ. For this reason, it is convenient to distinguish various parts of $R^{2}$. Before doing so, it is convenient to adopt several simplifying conventions. First, we set

$$
R_{\sigma}^{-}=(-\infty, \sigma], \quad R_{\sigma}^{+}=(\sigma, \infty) .
$$

Next, if $I$ and $J$ are real intervals, their cross-product $I \times J$ will be denoted by $I J$ instead, and we will assume that this product receives the priority attention in a mathematical expression. By means of these convections,

$$
\begin{array}{ll}
R_{\sigma}^{+} R_{\sigma}^{+}=(\sigma, \infty)^{2}, & R_{\sigma}^{+} R_{\sigma}^{-}=(\sigma, \infty) \times(-\infty, \sigma], \\
R_{\sigma}^{-} R_{\sigma}^{-}=(-\infty, \sigma]^{2}, & R_{\sigma}^{-} R_{\sigma}^{+}=(-\infty, \sigma] \times(\sigma, \infty) .
\end{array}
$$

Clearly, $\left\{R_{\sigma}^{+} R_{\sigma}^{+}, R_{\sigma}^{+} R_{\sigma}^{-}, R_{\sigma}^{-} R_{\sigma}^{+}, R_{\sigma}^{-} R_{\sigma}^{-}\right\}$is a partition of $\mathbf{R}^{2}$. Other subsets of the plane will be introduced in the subsequent sections.

For solutions originated from $z_{-2}$ and $z_{-1}$ in the above subsets, we will show that they are all "asymptotically periodic." More precisely, we say that a positive integer $\eta$ is a period of a scalar or vector sequence $v=\left\{v_{n}\right\}_{n=\alpha}^{\infty}$ if $v_{\eta+n}=v_{n}$ for all $n \geq \alpha$ and that $\tau$ is the least or prime period of $\left\{v_{n}\right\}_{n=\alpha}^{\infty}$ if $\tau$ is the least among all periods of $\left\{v_{n}\right\}_{n=\alpha}^{\infty}$. The sequence $\left\{v_{n}\right\}_{n=\alpha}^{\infty}$ is said to be $\tau$-periodic if $\tau$ is the least period of $\left\{v_{n}\right\}_{n=\alpha}^{\infty}$. The sequence $\left\{v_{n}\right\}_{n=\alpha}^{\infty}$ is said to be asymptotically periodic if there exist real numbers $v^{(0)}, v^{(1)}, \ldots, v^{(\omega-1)}$, where $\omega$ is a positive integer such that

$$
\lim _{n \rightarrow \infty} v_{\omega n+i}=v^{(i)}, \quad i=0,1, \ldots, \omega-1 .
$$

In case $\left\{v^{(0)}, v^{(1)}, \ldots, v^{(\omega-1)}, v^{(0)}, v^{(1)}, \ldots, v^{(\omega-1)}, \ldots\right\}$ is also an $\omega$-periodic sequence, we say that $v$ is an asymptotically $\omega$ periodic sequence tending to the limit $\omega$-cycle (this term is introduced since the underlying concept is similar to that of the limit cycle in the theory of ordinary differential equations) $\left\langle v^{(0)}, v^{(1)}, \ldots, v^{(\omega-1)}\right\rangle$. For the sake of convenience, for such an asymptotically $\omega$-periodic sequence $v$, we write

$$
v_{n} \longrightarrow\left\langle v^{(0)}, v^{(1)}, \ldots, v^{(\omega-1)}\right\rangle .
$$

Note that in case $v$ is asymptotically 1-periodic, then it is a sequence convergent to $v^{(0)}$ for some $v^{(0)}$. The converse is also true. In such a case, we simply write $v_{n} \rightarrow v^{(0)}$ instead of $v_{n} \rightarrow\left\langle v^{(0)}\right\rangle$.

Having these terminologies at hand, our main issue is to show that each solution of (3) originated from $\mathbf{R}^{2}$ is either asymptotically 1 - or 2 -periodic. Note, however, that since $f_{\sigma}$ is a discontinuous function, the standard theories that employ continuous arguments cannot be applied to yield asymptotic criteria. Fortunately, we may resort to elementary arguments as to be seen below.

Before doing so, let us make a few remarks. First note that our system (7) is autonomous (time invariant) and also symmetric in the sense that under two sets of "symmetric initial conditions," the behaviors of the corresponding solutions are also "symmetric." This statement can be made more precise in mathematical terms. However, a simple example is sufficient to illustrate this: if $\left\{z_{n}\right\}_{n=-2}^{\infty}$ is a solution of (3) with $\left(z_{-2}, z_{-1}\right) \in R_{\sigma}^{+} R_{\sigma}^{+} \times R_{\sigma}^{-} R_{\sigma}^{-}$, then as will be seen below, $z_{2 n} \rightarrow(1,-1)$ and $z_{2 n+1} \rightarrow(-1,1)$. If we now replace the condition $\left(z_{-2}, z_{-1}\right) \in R_{\sigma}^{+} R_{\sigma}^{+} \times R_{\sigma}^{-} R_{\sigma}^{-}$with the symmetric initial condition $\left(z_{-2}, z_{-1}\right) \in R_{\sigma}^{-} R_{\sigma}^{-} \times R_{\sigma}^{+} R_{\sigma}^{+}$, then we will end up with the conclusion that $z_{2 n} \rightarrow(-1,1)$ and $z_{2 n+1} \rightarrow$ $(1,-1)$. Such two conclusions will be referred to as dual results, and the principle of proof for either one can be applied to that of the other.

In the sequel, we will first distinguish three different cases (i) $0 \leq \sigma<1$, (ii) $\sigma=1$, and (iii) $\sigma>1$ and then consider different $z_{-2}, z_{-1}$ in $R_{\sigma}^{+} R_{\sigma}^{+}, R_{\sigma}^{+} R_{\sigma}^{-}, R_{\sigma}^{-} R_{\sigma}^{+}$, or $R_{\sigma}^{-} R_{\sigma}^{-}$ and the (asymptotic) behaviors of the corresponding solutions determined by them.

We will need the following simple but useful results. First, let $\left\{x_{k}\right\}_{k=-2}^{\infty}$ be real scalar (or vector) sequences that satisfy

$$
\begin{gathered}
x_{2 k}=a x_{2 k-2}+d, \quad n \in \mathbf{N}, \\
x_{2 k+1}=a x_{2 k-1}+d, \quad k \in \mathbf{N},
\end{gathered}
$$

where $a \in(0,1)$, and $d$ is a real number (resp., a real vector).

(i) If $\left\{x_{n}\right\}_{n=-2}^{\infty}$ is a sequence which satisfies (12), then

$$
x_{2 k}=a^{k+1} x_{-2}+\frac{\left(1-a^{k+1}\right)}{1-a} d, \quad k \in \mathbf{N} .
$$

(ii) If $\left\{x_{n}\right\}_{n=-2}^{\infty}$ is a sequence which satisfies (13), then

$$
x_{2 k+1}=a^{k+1} x_{-1}+\frac{\left(1-a^{k+1}\right)}{1-a} d, \quad k \in \mathbf{N} .
$$


Second, the function $F_{\sigma}$ in (6) satisfies

$$
\begin{array}{ll}
F_{\sigma}\left(R_{\sigma}^{+} R_{\sigma}^{+}\right)=-\overrightarrow{\mathbf{k}}, & F_{\sigma}\left(R_{\sigma}^{+} R_{\sigma}^{-}\right)=\overrightarrow{\mathbf{i}}, \\
F_{\sigma}\left(R_{\sigma}^{-} R_{\sigma}^{+}\right)=-\overrightarrow{\mathbf{i}}, & F_{\sigma}\left(R_{\sigma}^{-} R_{\sigma}^{-}\right)=\overrightarrow{\mathbf{k}},
\end{array}
$$

where $\overrightarrow{\mathbf{i}}=(1,-1)$ and $\overrightarrow{\mathbf{k}}=(1,1)$.

Third, we need to consider various ordering arrangements for three nonnegative integers $k, p$, and $l$ or four nonnegative integers $k, p, l$, and $m$. First, the ordering arrangements of three integers $k, p$, and $l$ can be classified into 6 cases:
(A1) $k=p \leq l$
(A2) $l=k<p$
(A3) $p=l<k$
(A4) $k<\min \{p, l\}$
(A5) $p<\min \{k, l\}$
(A6) $l<\min \{k, p\}$.

Indeed, let $a, b, c \in \mathbf{R}$. Then, since either $a<b, a=b$ or $a>b$, we see that

$$
\begin{gathered}
a<b \Longrightarrow c \in(-\infty, a), \\
c=a, c \in(a, b), c=b \text { or } c \in(b, \infty), \\
a=b \Longrightarrow c \in(-\infty, a), \quad c=a \text { or } c \in(a, \infty), \\
a>b \Longrightarrow c \in(-\infty, b), \\
c=b, c \in(b, a), c=a \text { or } c \in(a, \infty) .
\end{gathered}
$$

These are equivalent to

$$
\begin{aligned}
& a=b \leq c, \quad c=a<b, \quad b=c<a, \\
& a<\min \{b, c\}, \quad b<\min \{a, c\}, \quad c<\min \{a, b\},
\end{aligned}
$$

by comparing the two sets of statements (18) and (19).

By similar reasoning, there are 12 ordering arrangements for four nonnegative integers $k, p, l$, and $m$ :
(B1) $k=p \leq \min \{l, m\}$
(B2) $k<\min \{p, l, m\}$
(B3) $p<\min \{k, l, m\}$
(B4) $p=l<\min \{k, m\}$
(B5) $p=l=m<k$
(B6) $l<\min \{p, k, m\}$
(B7) $l=m<\min \{k, p\}$
(B8) $l=m=k<p$
(B9) $m<\min \{l, k, p\}$
(B10) $m=k<\min \{l, p\}$
(B11) $p=m<\min \{l, k\}$
(B12) $k=l<\min \{p, m\}$.

In the sequel, if $\alpha, \beta \in \mathbf{R}$ and $I, J$ are real intervals, then we adopt the convention that

$$
\begin{gathered}
\alpha I=\{\alpha x \mid x \in I\}, \quad \alpha+I=\{\alpha+x \mid x \in I\}, \\
\alpha I J=\{\alpha x \mid x \in I\} \times\{\alpha y \mid y \in J\}, \\
(\alpha, \beta)+I J=\{\alpha+x \mid x \in I\} \times\{\beta+y \mid y \in J\} .
\end{gathered}
$$

On the other hand, since $a \in(0,1), \sigma>0$, we have

$$
\begin{aligned}
\sigma & <\frac{\sigma+(1-a)}{a}<\frac{\sigma+\left(1-a^{2}\right)}{a^{2}}<\cdots<\frac{\sigma+\left(1-a^{k}\right)}{a^{k}} \\
& \longrightarrow+\infty .
\end{aligned}
$$

If we let

$$
\begin{gathered}
J^{(k)}=\left(\frac{\sigma+\left(1-a^{k}\right)}{a^{k}}, \frac{\sigma+\left(1-a^{k+1}\right)}{a^{k+1}}\right], \quad k \in \mathbf{N}, \\
J^{(-1)}=a J^{(0)}-a^{\prime}=(a \sigma-(1-a), \sigma],
\end{gathered}
$$

then

$$
\begin{gathered}
a J^{(k)}-a^{\prime}=J^{(k-1)}, \quad k \geq 0, \\
J^{(-1)} \subset R_{\sigma}^{-}, \quad R_{\sigma}^{+}=\bigcup_{k=0}^{\infty} J^{(k)}, \\
R_{\sigma}^{+} R_{\sigma}^{+}=\bigcup_{k, s=0}^{\infty} J^{(k)} J^{(s)}=\bigcup_{k=0}^{\infty} J^{(k)} R_{\sigma}^{+}=\bigcup_{s=0}^{\infty} R_{\sigma}^{+} J^{(s)} .
\end{gathered}
$$

\section{The Case $\sigma=1$}

In this section, we assume that $\sigma=1$.

Let $\left\{z_{n}\right\}_{n=-2}^{\infty}$ be a solution of (3). Let us consider first the case where $\left(z_{n}, z_{n+1}\right) \in R_{1}^{-} R_{1}^{-} \times R_{1}^{-} R_{1}^{-}$. Then by (7) and (16), we see that

$$
\begin{aligned}
& z_{n+2}=a z_{n}+a^{\prime} \overrightarrow{\mathbf{k}}, \\
& a R_{1}^{-} R_{1}^{-}+a^{\prime} F_{1}\left(R_{1}^{-} R_{1}^{-}\right)= a R_{1}^{-} R_{1}^{-}+a^{\prime} \overrightarrow{\mathbf{k}} \\
&= a((-\infty, 1] \times(-\infty, 1]) \\
&+(1-a)(1,1) \\
&=(-\infty, 1] \times(-\infty, 1] \\
&= R_{1}^{-} R_{1}^{-} .
\end{aligned}
$$

Hence, we may see further that $z_{n+2} \in R_{1}^{-} R_{1}^{-}$. Similarly, since

$$
\begin{aligned}
a J^{(k)} R_{1}^{-}+a^{\prime} F_{1}\left(R_{1}^{-} J^{(s)}\right) & =a J^{(k)} R_{1}^{-}+a^{\prime}(-\overrightarrow{\mathbf{i}}) \\
& =a\left(J^{(k)} \times(-\infty, 1]\right)+(1-a)(-1,1) \\
& =J^{(k-1)} \times(-\infty, \sigma] \\
& =J^{(k-1)} R_{1}^{-},
\end{aligned}
$$

we see that if $\left(z_{n}, z_{n+1}\right) \in J^{(k)} R_{1}^{-} \times R_{1}^{-} J^{(s)}$, then $z_{n+2} \in J^{(k-1)} R_{1}^{-}$. By similar considerations, we may build a "directed graph" represented by Table 1 .

In this table, we record the fact that $a R_{1}^{-} R_{1}^{-}+a^{\prime} F_{1}\left(R_{1}^{-} R_{1}^{-}\right) \subseteq$ $R_{1}^{-} R_{1}^{-}$as the $\left(R_{1}^{-} R_{1}^{-}, R_{1}^{-} R_{1}^{-}\right)$entry and so forth.

Next, we let $\left\{z_{n}\right\}_{n=-2}^{\infty}$ be a solution of (3) again. If $\left(z_{-2}, z_{-1}\right) \in R_{1}^{-} R_{1}^{-} \times R_{1}^{-} R_{1}^{-}$, then from Table 1 we see that $z_{0} \in R_{1}^{-} R_{1}^{-}$. Now that $\left(z_{-1}, z_{0}\right) \in R_{1}^{-} R_{1}^{-} \times R_{1}^{-} R_{1}^{-}$, we may use Table 1 to infer that $z_{1} \in R_{1}^{-} R_{1}^{-}$again. By induction, we see that $z_{n} \in R_{1}^{-} R_{1}^{-}$for all $n \geq-2$. By (7), we then see that

$$
z_{n}=a z_{n-2}+a^{\prime} \overrightarrow{\mathbf{k}}, \quad n \in \mathbf{N},
$$


and hence by (14) and (15), we see that $z_{n} \rightarrow \overrightarrow{\mathbf{k}}$ as $n \rightarrow \infty$. If $\left(z_{-2}, z_{-1}\right) \in R_{1}^{-} R_{1}^{+} \times R_{1}^{-} R_{1}^{-}$, then from the $\left(R_{1}^{-} R_{1}^{+}, R_{1}^{-} R_{1}^{-}\right)$ entry of Table 1 we see that $z_{0} \in R_{1}^{-} R_{1}^{+}$. Now that $\left(z_{-1}, z_{0}\right) \in$ $R_{1}^{-} R_{1}^{-} \times R_{1}^{-} R_{1}^{+}$, by Table 1 again, we see that

$$
z_{1}=a z_{-1}+a^{\prime}(-\overrightarrow{\mathbf{i}}) \in R_{1}^{-} R_{1}^{-} .
$$

By induction, we see that $z_{2 k} \in R_{1}^{-} R_{1}^{+}$and $z_{2 k+1} \in R_{1}^{-} R_{1}^{-}$for $k \geq-1$. By (7), we may see further that

$$
\begin{gathered}
z_{2 n}=a z_{2 n-2}+a^{\prime} \overrightarrow{\mathbf{k}}, \\
z_{2 n+1}=a z_{2 n-1}-a^{\prime} \overrightarrow{\mathbf{i}},
\end{gathered}
$$

for $n \in \mathbf{N}$, and hence by (14) and (15), $z_{2 n} \rightarrow \overrightarrow{\mathbf{k}}$ and $z_{2 n+1} \rightarrow$ $-\overrightarrow{\mathbf{i}}$ as $n \rightarrow \infty$. By considering $z_{-2}$ and $z_{-1}$ in different parts of the plane, we may apply the same principle to obtain Table 2.

In this table, there are seven indeterminate cases. Let us go through two cases. First, let $\left(z_{-2}, z_{-1}\right) \in R_{1}^{+} R_{1}^{-} \times R_{1}^{-} R_{1}^{+}$. Then in view of (25), $\left(z_{-2}, z_{-1}\right) \in J^{(k)} R_{1}^{-} \times R_{1}^{-} J^{(s)}$ for some $k, s \in \mathbf{N}$. There are then two subcases: (i) $0 \leq k \leq s$ or (ii) $0 \leq s<k$. In the former case, by Table 1 ,

$$
z_{0}=a z_{-2}+a^{\prime} F_{1}\left(z_{-1}\right) \in J^{(k-1)} R_{1}^{-},
$$

and (hence)

$$
z_{1}=a z_{-1}+a^{\prime} F_{1}\left(z_{0}\right) \in R_{1}^{-} J^{(s-1)},
$$

and by induction,

$$
\begin{gathered}
z_{2 k} \in J^{(-1)} R_{1}^{-} \subseteq R_{1}^{-} R_{1}^{-}, \\
z_{2 k+1} \in R_{1}^{-} R_{1}^{+} .
\end{gathered}
$$

In the latter case, we may similarly show that $\left(z_{2 s}, z_{2 s+1}\right) \epsilon$ $R_{1}^{+} R_{1}^{-} \times R_{1}^{-} R_{1}^{-}$. By considering $\left(z_{-2}, z_{-1}\right)$ in different parts of the plane, we may then construct the following three selfexplanatory Tables 3,4 , and 5 .

As a consequence, if $\left(z_{-2}, z_{-1}\right) \in J^{(k)} R_{1}^{-} \times R_{1}^{-} J^{(s)}$ and $0 \leq$ $k \leq s$, then the solution of (3) originated from $z_{-2}$ and $z_{-1}$ will satisfy $\left(z_{2 k}, z_{2 k+1}\right) \in R_{1}^{-} R_{1}^{-} \times R_{1}^{-} R_{1}^{+}$. Then by Table $2, z_{n} \rightarrow$ $\langle-\overrightarrow{\mathbf{i}}, \overrightarrow{\mathbf{k}}\rangle$.

Next, let $\left(z_{-2}, z_{-1}\right) \in R_{1}^{+} R_{1}^{+} \times R_{1}^{+} R_{1}^{+}$. Then by (25), $\left(z_{-2}\right.$, $\left.z_{-1}\right) \in J^{(k)} J^{(p)} \times J^{(l)} J^{(m)}$ for some $k, p, l, m \in \mathbf{N}$. As mentioned before, there are twelve different ordering arrangements B1-B12 for these integers. In case B1 holds, we may make use of Table 1 repeatedly to show that $\left(z_{2 k}, z_{2 k+1}\right) \in$ $R_{1}^{-} R_{1}^{-} \times R_{1}^{+} R_{1}^{+}$(which is recorded in self-explanatory Table 6), and then by Table 2 , we finally see that $z_{n} \rightarrow\langle-\overrightarrow{\mathbf{k}}, \overrightarrow{\mathbf{k}}\rangle$.

In conclusion, we have shown the following result.

Theorem 1. Suppose that $\sigma=1$. Then, a solution of (3) must either be asymptotically 1-periodic tending to the limit cycles

$$
\langle\overrightarrow{\mathbf{i}}\rangle,\langle-\overrightarrow{\mathbf{i}}\rangle, \text { or }\langle\overrightarrow{\mathbf{k}}\rangle,
$$

or asymptotically 2-periodic tending to the limit cycles

$$
\langle\overrightarrow{\mathbf{i}}, \overrightarrow{\mathbf{k}}\rangle, \quad\langle-\overrightarrow{\mathbf{i}}, \overrightarrow{\mathbf{k}}\rangle, \quad \text { or } \quad\langle-\overrightarrow{\mathbf{k}}, \overrightarrow{\mathbf{k}}\rangle \text {. }
$$

TABLE 1

\begin{tabular}{lcccccc}
\hline & $R_{1}^{-} R_{1}^{-}$ & $J^{(s)} R_{1}^{-}$ & $R_{1}^{-} J^{(s)}$ & $R_{1}^{+} J^{(s)}$ & $J^{(s)} R_{1}^{+}$ & $J^{(s)} J^{(l)}$ \\
\hline$R_{1}^{-} R_{1}^{-}$ & $R_{1}^{-} R_{1}^{-}$ & $R_{1}^{-} R_{1}^{-}$ & $R_{1}^{-} R_{1}^{-}$ & $R_{1}^{-} R_{1}^{-}$ & $R_{1}^{-} R_{1}^{-}$ & $R_{1}^{-} R_{1}^{-}$ \\
$J^{(k)} R_{1}^{-}$ & $R_{1}^{+} R_{1}^{-}$ & $R_{1}^{+} R_{1}^{-}$ & $J^{(k-1)} R_{1}^{-}$ & $J^{(k-1)} R_{1}^{-}$ & & \\
$R_{1}^{-} J^{(k)}$ & $R_{1}^{-} R_{1}^{+}$ & $R_{1}^{-} J^{(k-1)}$ & $R_{1}^{-} R_{1}^{+}$ & & $R_{1}^{-} J^{(k-1)}$ & \\
$R_{1}^{+} J^{(k)}$ & $R_{1}^{+} R_{1}^{+}$ & $R_{1}^{+} J^{(k-1)}$ & & & & \\
$J^{(k)} R_{1}^{+}$ & $R_{1}^{+} R_{1}^{+}$ & & $J^{(k-1)} R_{1}^{+}$ & & & \\
$J^{(k)} J^{(p)}$ & $R_{1}^{+} R_{1}^{+}$ & & & & & $J^{(k-1)} J^{(p-1)}$ \\
\hline
\end{tabular}

TABLE 2

\begin{tabular}{ccccc}
\hline & $R_{1}^{-} R_{1}^{-}$ & $R_{1}^{+} R_{1}^{-}$ & $R_{1}^{-} R_{1}^{+}$ & $R_{1}^{+} R_{1}^{+}$ \\
\hline$R_{1}^{-} R_{1}^{-}$ & $\langle\overrightarrow{\mathbf{k}}\rangle$ & $\langle\overrightarrow{\mathbf{i}}, \overrightarrow{\mathbf{k}}\rangle$ & $\langle-\overrightarrow{\mathbf{i}}, \overrightarrow{\mathbf{k}}\rangle$ & $\langle-\overrightarrow{\mathbf{k}}, \overrightarrow{\mathbf{k}}\rangle$ \\
$R_{1}^{+} R_{1}^{-}$ & $\langle\overrightarrow{\mathbf{k}}, \overrightarrow{\mathbf{i}}\rangle$ & $\langle\overrightarrow{\mathbf{i}}\rangle$ & See Table 3 & See Table 4 \\
$R_{1}^{-} R_{1}^{+}$ & $\langle\overrightarrow{\mathbf{k}},-\overrightarrow{\mathbf{i}}\rangle$ & See Table 3 & $\langle-\overrightarrow{\mathbf{i}}\rangle$ & See Table 4 \\
$R_{1}^{+} R_{1}^{+}$ & $\langle\overrightarrow{\mathbf{k}},-\overrightarrow{\mathbf{k}}\rangle$ & See Table 5 & See Table 5 & See Table 6 \\
\hline
\end{tabular}

TABLE 3

\begin{tabular}{ll}
\hline$\left(z_{-2}, z_{-1}\right) \in J^{(k)} R^{-} \times R_{1}^{-} J^{(s)}$ & $\left(R_{1}^{-} J^{(k)} \times J^{(s)} R_{1}^{-}\right)$ \\
\hline $0 \leq k \leq s \Rightarrow\left(z_{2 k}, z_{2 k+1}\right) \in R_{1}^{-} R_{1}^{-} \times R_{1}^{-} R_{1}^{+}$ & $\left(R_{1}^{-} R_{1}^{-} \times R_{1}^{+} R_{1}^{-}\right)$ \\
$0 \leq s<k \Rightarrow\left(z_{2 s}, z_{2 s+1}\right) \in R_{1}^{+} R_{1}^{-} \times R_{1}^{-} R_{1}^{-}$ & $\left(R_{1}^{-} R_{1}^{+} \times R_{1}^{-} R_{1}^{-}\right)$ \\
\hline
\end{tabular}

TABLE 4

\begin{tabular}{ll}
\hline$\left(z_{-2}, z_{-1}\right) \in J^{(k)} R^{-} \times R_{1}^{+} J^{(s)}$ & $\left(R_{1}^{-} J^{(k)} \times J^{(s)} R_{1}^{+}\right)$ \\
\hline $0 \leq k \leq s \Rightarrow\left(z_{2 k}, z_{2 k+1}\right) \in R_{1}^{-} R_{1}^{-} \times R_{1}^{+} R_{1}^{+}$ & $\left(R_{1}^{-} R_{1}^{-} \times R_{1}^{+} R_{1}^{+}\right)$ \\
$0 \leq s<k \Rightarrow\left(z_{2 s}, z_{2 s+1}\right) \in R_{1}^{+} R_{1}^{-} \times R_{1}^{+} R_{1}^{-}$ & $\left(R_{1}^{-} R_{1}^{+} \times R_{1}^{-} R_{1}^{+}\right)$ \\
\hline
\end{tabular}

We remark that, as can be seen from the above exhaustive arguments, the "region of attraction" of each limit cycle can be given, here if $S$ is the set of all solutions of (3) that tend to the limit cycle $G$, then $\left\{\left(z_{-2}, z_{-1}\right) \in R^{2} \times R^{2} \mid\left\{z_{k}\right\}_{k=-2}^{\infty} \in S\right\}$ is called the region of attraction of $G$.

Example 2. Consider the region of attraction of the limit cycle $\langle\overrightarrow{\mathbf{i}}, \overrightarrow{\mathbf{k}}\rangle$. By Table 2, we first see that solutions with initial values in $R_{1}^{-} R_{1}^{-} \times R_{1}^{+} R_{1}^{-}$or $R_{1}^{+} R_{1}^{-} \times R_{1}^{-} R_{1}^{-}$tend to the limit cycle $\langle\overrightarrow{\mathbf{i}}, \overrightarrow{\mathbf{k}}\rangle$ (and hence $\langle\overrightarrow{\mathbf{k}}, \overrightarrow{\mathbf{i}}\rangle$ ); and solutions with initial values in the seven indeterminate cases may or may not tend to the limit cycle $\langle\overrightarrow{\mathbf{i}}, \overrightarrow{\mathbf{k}}\rangle$.

Therefore, we next consider Table 3. If $\left(z_{-2}, z_{-1}\right) \in$ $J^{(k)} R_{1}^{-} \times R_{1}^{-} J^{(s)}$ where $0 \leq s<k$, then $\left(z_{2 s}, z_{2 s+1}\right) \in R_{1}^{+} R_{1}^{-} \times$ $R_{1}^{-} R_{1}^{-}$and hence $z_{n} \rightarrow\langle\overrightarrow{\mathbf{k}}, \overrightarrow{\mathbf{i}}\rangle$. If $\left(z_{-2}, z_{-1}\right) \in R_{1}^{-} J^{(k)} \times J^{(s)} R_{1}^{-}$ where $0 \leq k \leq s$, then $\left(z_{2 k}, z_{2 k+1}\right) \in R_{1}^{-} R_{1}^{-} \times R_{1}^{+} R_{1}^{-}$and hence $z_{n} \rightarrow\langle\overrightarrow{\mathbf{i}}, \overrightarrow{\mathbf{k}}\rangle$. Furthermore, no other cases in Tables 3, 4, and 5 can lead to $\left(z_{m}, z_{m+1}\right) \in R_{1}^{+} R_{1}^{-} \times R_{1}^{+} R_{1}^{-}, R_{1}^{-} R_{1}^{+} \times R_{1}^{-} R_{1}^{+}$, or $R_{1}^{+} R_{1}^{+} \times R_{1}^{-} R_{1}^{-}$.

Next, we consider Table 6. By direct examination, there is no case which leads to $\left(x_{m}, x_{m+1}\right) \in R_{1}^{+} R_{1}^{-} \times R_{1}^{-} R_{1}^{-}$or $R_{1}^{-} R_{1}^{-} \times R_{1}^{+} R_{1}^{-}$. There is no case, except possibly $\mathrm{B} 10$, that leads 
TABLE 5

\begin{tabular}{ll}
\hline$\left(z_{-2}, z_{-1}\right) \in R_{1}^{+} J^{(k)} \times J^{(s)} R_{1}^{-}$ & $\left(J^{(k)} R_{1}^{+} \times R_{1}^{-} J^{(s)}\right)$ \\
\hline $0 \leq k \leq s \Rightarrow\left(z_{2 k}, z_{2 k+1}\right) \in R_{1}^{+} R_{1}^{-} \times R_{1}^{+} R_{1}^{-}$ & $\left(R_{1}^{-} R_{1}^{+} \times R_{1}^{-} R_{1}^{+}\right)$ \\
$0 \leq s<k \Rightarrow\left(z_{2 s}, z_{2 s+1}\right) \in R_{1}^{+} R_{1}^{+} \times R_{1}^{-} R_{1}^{-}$ & $\left(R_{1}^{+} R_{1}^{+} \times R_{1}^{-} R_{1}^{-}\right)$ \\
\hline
\end{tabular}

TABLE 6

\begin{tabular}{l}
$\overline{\left(z_{-2}, z_{-1}\right) \in J^{(k)} J^{(p)} \times J^{(l)} J^{(m)}}$ \\
\hline $\mathrm{B} 1 \Rightarrow\left(z_{2 k}, z_{2 k+1}\right) \in R_{1}^{-} R_{1}^{-} \times R_{1}^{+} R_{1}^{+}$ \\
$\mathrm{B} 2 \Rightarrow\left(z_{2 k}, z_{2 k+1}\right) \in R_{1}^{-} J^{(p-k-1)} \times J^{(l-k-1)} R_{1}^{+}$ \\
$\mathrm{B} 3 \Rightarrow\left(z_{2 p}, z_{2 p+1}\right) \in J^{(k-p-1)} R_{1}^{-} \times R_{1}^{+} J^{(m-p-1)}$ \\
$\mathrm{B} 4 \Rightarrow\left(z_{2 p}, z_{2 p+1}\right) \in J^{(k-p-1)} R_{1}^{-} \times R_{1}^{+} J^{(m-p-1)}$ \\
$\mathrm{B} 5 \Rightarrow\left(z_{2 p}, z_{2 p+1}\right) \in R_{1}^{+} R_{1}^{-} \times R_{1}^{+} R_{1}^{-}$ \\
$\mathrm{B} 6 \Rightarrow\left(z_{2 l}, z_{2 l+1}\right) \in J^{(k-l-1)} R_{1}^{+} \times R_{1}^{-} J^{(m-l-1)}$ \\
$\mathrm{B} 7 \Rightarrow\left(z_{2 l}, z_{2 l+1}\right) \in R_{1}^{+} R_{1}^{+} \times R_{1}^{-} R_{1}^{-}$ \\
$\mathrm{B} 8 \Rightarrow\left(z_{2 l}, z_{2 l+1}\right) \in R_{1}^{-} R_{1}^{+} \times R_{1}^{-} R_{1}^{+}$ \\
$\mathrm{B} 9 \Rightarrow\left(z_{2 m}, z_{2 m+1}\right) \in R_{1}^{+} J^{(p-m-1)} \times J^{(l-m-1)} R_{1}^{-}$ \\
$\mathrm{B} 10 \Rightarrow\left(z_{2 k}, z_{2 k+1}\right) \in R_{1}^{-} J^{(p-k-1)} \times J^{(l-k-1)} R_{1}^{+}$ \\
$\mathrm{B} 11 \Rightarrow\left(z_{2 p}, z_{2 p+1}\right) \in R_{1}^{+} R_{1}^{-} \times R_{1}^{+} R_{1}^{-}$ \\
$\mathrm{B} 12 \Rightarrow\left(z_{2 l}, z_{2 l+1}\right) \in R_{1}^{-} R_{1}^{+} \times R_{1}^{-} R_{1}^{+}$ \\
\hline
\end{tabular}

to $\left(x_{m}, x_{m+1}\right) \in J^{(k)} R_{1}^{-} \times R_{1}^{-} J^{(s)}$ with $0 \leq s<k$, or $\left(z_{-2}, z_{-1}\right) \epsilon$ $R_{1}^{-} J^{(k)} \times J^{(s)} R_{1}^{-}$with $0 \leq k \leq s$. However, if B10 holds and $p-k-1 \leq l-k-1$, then $\left(z_{-2}, z_{-1}\right) \in J^{(k)} J^{(p)} \times J^{(l)} J^{(m)}$ implies $\left(z_{2 k}, z_{2 k+1}\right) \in R_{1}^{-} J^{(p-k-1)} \times J^{(l-k-1)} R_{1}^{+}$, which in turn implies (by Table 3 ) that $\left(z_{2 p}, z_{2 p+1}\right) \in R_{1}^{-} R_{1}^{-} \times R_{1}^{+} R_{1}^{-}$. Hence, $z_{n} \rightarrow\langle\overrightarrow{\mathbf{i}}, \overrightarrow{\mathbf{k}}\rangle$.

In summary, the region of attraction of $\langle\overrightarrow{\mathbf{i}}, \overrightarrow{\mathbf{k}}\rangle$ is the union of the following sets:

$$
\begin{aligned}
& R_{1}^{-} R_{1}^{-} \times R_{1}^{+} R_{1}^{-}, R_{1}^{+} R_{1}^{-} \times R_{1}^{-} R_{1}^{-}, \\
& R_{1}^{-} J^{(k)} \times J^{(s)} R_{1}^{-}(0 \leq k \leq s), \\
& J^{(k)} R_{1}^{+} \times R_{1}^{-} J^{(s)}(0 \leq s<k), \\
& J^{(k)} J^{(P)} \times J^{(l)} J^{(m)} \quad(m=k<\min \{l, p\}, \\
&p-k-1 \leq l-k-1) .
\end{aligned}
$$

\section{The Case $\sigma>1$}

In this section, we assume that $\sigma>1$.

Lemma 3. Suppose that $\sigma>1$. Let $\left\{z_{n}\right\}_{n=-2}^{\infty}$ be a solution of (3) originated from $z_{-2}, z_{-1} \in R_{\sigma}^{+} R_{\sigma}^{+}$. Then, there exists $m \in$ $\{-1,0, \ldots\}$ such that $z_{m} \in R_{\sigma}^{+} R_{\sigma}^{+}$and $z_{m+1} \in \mathbf{R}^{2} \backslash R_{\sigma}^{+} R_{\sigma}^{+}$.

Proof. Let $\left\{z_{n}\right\}_{n=-2}^{\infty}$ be a solution of (3) originated from $z_{-2}, z_{-1} \in R_{\sigma}^{+} R_{\sigma}^{+}$. Assume to the contrary that $z_{n} \in R_{\sigma}^{+} R_{\sigma}^{+}$for all $n \in \mathbf{N}$. Then,

$$
z_{n}=a z_{n-2}+a^{\prime}(-\overrightarrow{\mathbf{k}}), \quad n \in \mathbf{N} .
$$

By (14) and (15), we see that $z_{n} \rightarrow-\overrightarrow{\mathbf{k}}$ as $n \rightarrow \infty$. But $\sigma>1$ implies $-\overrightarrow{\mathbf{k}}$ is in the interior of $R_{\sigma}^{-} R_{\sigma}^{-}$which is disjointed from $R_{\sigma}^{+} R_{\sigma}^{+}$. A contradiction is, thus, obtained.
TABLE 7

\begin{tabular}{lcccc}
\hline & $R_{\sigma}^{-} R_{\sigma}^{-}$ & $I^{(s)} R_{\sigma}^{-}$ & $J^{(s)} R_{\sigma}^{-}$ & $R_{\sigma}^{-} I^{(s)}$ \\
\hline$R_{\sigma}^{-} R_{\sigma}^{-}$ & $R_{\sigma}^{-} R_{\sigma}^{-}$ & $R_{\sigma}^{-} R_{\sigma}^{-}$ & $R_{\sigma}^{-} R_{\sigma}^{-}$ & $R_{\sigma}^{-} R_{\sigma}^{-}$ \\
$I^{(k)} R_{\sigma}^{-}$ & $I^{(k-1)} R_{\sigma}^{-}$ & $I^{(k-1)} R_{\sigma}^{-}$ & $I^{(k-1)} R_{\sigma}^{-}$ & \\
$J^{(k)} R_{\sigma}^{-}$ & & & & $J^{(k-1)} R_{\sigma}^{-}$ \\
$R_{\sigma}^{-} I^{(k)}$ & $R_{\sigma}^{-} I^{(k-1)}$ & & & $R_{\sigma}^{-} I^{(k-1)}$ \\
$R_{\sigma}^{-} J^{(k)}$ & & $R_{\sigma}^{-} J^{(k-1)}$ & $R_{\sigma}^{-} J^{(k-1)}$ & \\
$I^{(k)} J^{(p)}$ & & $I^{(k-1)} J^{(p-1)}$ & $I^{(k-1)} J^{(p-1)}$ & \\
$J^{(k)} I^{(p)}$ & & & & $J^{(k-1)} I^{(p-1)}$ \\
$I^{(k)} I^{(p)}$ & $I^{(k-1)} I^{(p-1)}$ & & & \\
$J^{(k)} J^{(p)}$ & & & & \\
\hline
\end{tabular}

In view of Lemma 3, we only need to investigate the asymptotic behaviors of solutions $\left\{z_{n}\right\}_{n=-2}^{\infty}$ of (3) such that $z_{-2}$ and $z_{-1}$ are not simultaneously in $R_{\sigma}^{+} R_{\sigma}^{+}$. To this end, we first note that

$$
\sigma<\frac{\sigma \pm(1-a)}{a}<\frac{\sigma \pm\left(1-a^{2}\right)}{a^{2}}<\cdots<\frac{\sigma \pm\left(1-a^{k}\right)}{a^{k}}
$$

$\longrightarrow+\infty$.

Hence, if we let

$$
\begin{gathered}
I^{(k)}=\left(\frac{\sigma-\left(1-a^{k}\right)}{a^{k}}, \frac{\sigma-\left(1-a^{k+1}\right)}{a^{k+1}}\right], \quad k \in \mathbf{N}, \\
I^{(-1)}=a I^{(0)}+a^{\prime}=(a \sigma+(1-a), \sigma],
\end{gathered}
$$

then

$$
\begin{gathered}
a I^{(k)}+a^{\prime}=I^{(k-1)}, \quad k \in \mathbf{N}, \\
R_{\sigma}^{+}=\bigcup_{k=0}^{\infty} J^{(k)}=\bigcup_{k=0}^{\infty} I^{(k)}, \\
R_{\sigma}^{+} R_{\sigma}^{-}=\bigcup_{k=0}^{\infty} I^{(k)} R_{\sigma}^{-}=\bigcup_{k=0}^{\infty} J^{(k)} R_{\sigma}^{-}, \\
R_{\sigma}^{-} R_{\sigma}^{+}=\bigcup_{k=0}^{\infty} R_{\sigma}^{-} I^{(k)}=\bigcup_{k=0}^{\infty} R_{\sigma}^{-} J^{(k)}, \\
R_{\sigma}^{+} R_{\sigma}^{+}=\bigcup_{k, s=0}^{\infty} I^{(k)} J^{(s)}=\bigcup_{k, s=0}^{\infty} J^{(k)} I^{(s)} \\
=\bigcup_{k, s=0}^{\infty} I^{(k)} I^{(s)}=\bigcup_{k, s=0}^{\infty} J^{(k)} J^{(s)}, \\
I^{(-1)}, J^{(-1)} \subset R_{\sigma}^{-} .
\end{gathered}
$$

As in the previous section, we may build a table (consisting of two separate parts Tables 7 and 8) which plays the same role as Table 1.

Then, we may construct Tables 9-14 which play the same role as Tables 3-6.

Then by Lemma 3 and these tables, we may show the following result. 
TABLE 8

\begin{tabular}{lccccc}
\hline & $R_{\sigma}^{-} J^{(s)}$ & $I^{(s)} J^{(l)}$ & $J^{(s)} I^{(l)}$ & $I^{(s)} I^{(l)}$ & $J^{(s)} J^{(l)}$ \\
\hline$R_{\sigma}^{-} R_{\sigma}^{-}$ & $R_{\sigma}^{-} R_{\sigma}^{-}$ & $R_{\sigma}^{-} R_{\sigma}^{-}$ & $R_{\sigma}^{-} R_{\sigma}^{-}$ & $R_{\sigma}^{-} R_{\sigma}^{-}$ & $R_{\sigma}^{-} R_{\sigma}^{-}$ \\
$I^{(k)} R_{\sigma}^{-}$ & & & & & \\
$J^{(k)} R_{\sigma}^{-}$ & $J^{(k-1)} R_{\sigma}^{-}$ & $J^{(k-1)} R_{\sigma}^{-}$ & $J^{(k-1)} R_{\sigma}^{-}$ & $J^{(k-1)} R_{\sigma}^{-}$ & $J^{(k-1)} R_{\sigma}^{-}$ \\
$R_{\sigma}^{-} I^{(k)}$ & $R_{\sigma}^{-} I^{(k-1)}$ & & & & \\
$R_{\sigma}^{-} J^{(k)}$ & & $R_{\sigma}^{-} J^{(k-1)}$ & $R_{\sigma}^{-} J^{(k-1)}$ & $R_{\sigma}^{-} J^{(k-1)}$ & $R_{\sigma}^{-} J^{(k-1)}$ \\
$I^{(k)} J^{(p)}$ & & & & & \\
$J^{(k)} I^{(p)}$ & $J^{(k-1)} I^{(p-1)}$ & & & & \\
$I^{(k)} I^{(p)}$ & & & & & \\
$J^{(k)} J^{(p)}$ & & $J^{(k-1)} J^{(p-1)}$ & $J^{(k-1)} J^{(p-1)}$ & $J^{(k-1)} J^{(p-1)}$ & $J^{(k-1)} J^{(p-1)}$ \\
\hline
\end{tabular}

TABLE 9

$\overline{\left(z_{-2}, z_{-1}\right) \in R_{\sigma}^{-} R_{\sigma}^{-} \times I^{(k)} R_{\sigma}^{-} \Rightarrow\left(z_{2 k}, z_{2 k+1}\right) \in R_{\sigma}^{-} R_{\sigma}^{-} \times R_{\sigma}^{-} R_{\sigma}^{-}}$
$\left(z_{-2}, z_{-1}\right) \in R_{\sigma}^{-} R_{\sigma}^{-} \times R_{\sigma}^{-} I^{(k)} \Rightarrow\left(z_{2 k}, z_{2 k+1}\right) \in R_{\sigma}^{-} R_{\sigma}^{-} \times R_{\sigma}^{-} R_{\sigma}^{-}$
$\left(z_{-2}, z_{-1}\right) \in I^{(k)} R_{\sigma}^{-} \times R_{\sigma}^{-} R_{\sigma}^{-} \Rightarrow\left(z_{2 k}, z_{2 k+1}\right) \in R_{\sigma}^{-} R_{\sigma}^{-} \times R_{\sigma}^{-} R_{\sigma}^{-}$
$\left(z_{-2}, z_{-1}\right) \in R_{\sigma}^{-} I^{(k)} \times R_{\sigma}^{-} R_{\sigma}^{-} \Rightarrow\left(z_{2 k}, z_{2 k+1}\right) \in R_{\sigma}^{-} R_{\sigma}^{-} \times R_{\sigma}^{-} R_{\sigma}^{-}$

Lemma 4. Suppose that $\sigma>1$. Let $\left\{z_{n}\right\}_{n=-2}^{\infty}$ be a solution of (3). Then, there exists $m \in\{-2,-1, \ldots\}$ such that $z_{m}, z_{m+1} \in$ $R_{\sigma}^{-} R_{\sigma}^{-}$.

Proof. In view of Lemma 3, we may assume that $z_{-2} \in \mathbf{R}^{2}$ and $z_{-1} \in \mathbf{R}^{2} \backslash R_{\sigma}^{+} R_{\sigma}^{+}$, or, $z_{-2} \in \mathbf{R}^{2} \backslash R_{\sigma}^{+} R_{\sigma}^{+}$and $z_{-1} \in R_{\sigma}^{+} R_{\sigma}^{+}$.

Consider first the case where $z_{-2} \in R_{\sigma}^{+} R_{\sigma}^{-}$and $z_{-1} \in R_{\sigma}^{-} R_{\sigma}^{+}$. Then, $z_{-2} \in J^{(k)} R_{\sigma}^{-}$and $z_{-1} \in R_{\sigma}^{-} J^{(s)}$ for some $k, s \in \mathbf{N}$. By Table 11, if $0 \leq k \leq s$, then $\left(z_{2 k-1}, z_{2 k}\right) \in R_{\sigma}^{-} J^{(s-k)} \times R_{\sigma}^{-} R_{\sigma}^{-}$. Since $J^{(s-k)} \subset(\sigma,+\infty)$, there exists some $t \in \mathbf{N}$ such that $\left(z_{2 k-1}, z_{2 k}\right) \in R_{\sigma}^{-} I^{(t)} \times R_{\sigma}^{-} R_{\sigma}^{-}$. By the last entry in Table 9, we see that there is some $m$ such that $z_{m}, z_{m+1} \in R_{\sigma}^{-} R_{\sigma}^{-}$. If $0 \leq s<k$, then $\left(z_{2 s}, z_{2 s+1}\right) \in J^{(k-s-1)} R_{\sigma}^{-} \times R_{\sigma}^{-} R_{\sigma}^{-}$. Since $J^{(k-s-1)} \subset(\sigma,+\infty)$, there exists some $t \in \mathrm{N}$ such that $\left(z_{2 s}, z_{2 s+1}\right) \in I^{(t)} R_{\sigma}^{-} \times R_{\sigma}^{-} R_{\sigma}^{-}$. By the last entry in Table 9, we see that there is some $m$ such that $z_{m}, z_{m+1} \in R_{\sigma}^{-} R_{\sigma}^{-}$.

Consider next the case where $z_{-2} \in R_{\sigma}^{-} R_{\sigma}^{-}$and $z_{-1} \in$ $R_{\sigma}^{+} R_{\sigma}^{+}$. Then, $z_{-1} \in I^{(s)} I^{(k)}$ for some $k, s \in \mathbf{N}$. By Table 12 if $0 \leq k \leq s$, then $\left(z_{2 k}, z_{2 k+1}\right) \in R_{\sigma}^{-} R_{\sigma}^{-} \times I^{(s-k-1)} R_{\sigma}^{-}$. By the last entry in Table 9, we see that there is some $m$ such that $z_{m}, z_{m+1} \in R_{\sigma}^{-} R_{\sigma}^{-}$. If $0 \leq s<k$, then $\left(z_{2 s}, z_{2 s+1}\right) \in$ $R_{\sigma}^{-} R_{\sigma}^{-} \times R_{\sigma}^{-} I^{(k-s-1)}$. By the last entry in Table 9 , we see that there is some $m$ such that $\left(z_{m}, z_{m+1}\right) \in R_{\sigma}^{-} R_{\sigma}^{-}$.

Consider next the case where $z_{-2} \in R_{\sigma}^{+} R_{\sigma}^{-}$and $z_{-1} \in$ $R_{\sigma}^{+} R_{\sigma}^{+}$. Then, $z_{-2} \in J^{(k)} R_{\sigma}^{-}$and $z_{-1} \in I^{(l)} J^{(p)}$ for some $k, l, p \in$ N. By Table 13, if A1 holds, that is, $0 \leq k=p \leq l$, then $\left(z_{2 k-1}, z_{2 k}\right) \in I^{(l-k)} J^{(p-k)} \times R_{\sigma}^{-} R_{\sigma}^{-}$. Then, there exists $t \in \mathbf{N}$ such that $\left(z_{2 k-1}, z_{2 k}\right) \in I^{(l-k)} I^{(t)} \times R_{\sigma}^{-} R_{\sigma}^{-}$. By Table 12 again, if $0 \leq t \leq l-k$, then $\left(z_{2 t+2 k+1}, z_{2 t+2 k+2}\right) \in I^{(l-k-t-1)} R_{\sigma}^{-} \times R_{\sigma}^{-} R_{\sigma}^{-}$. By the last entry in Table 9, we see that there is some $m$ such that $z_{m}, z_{m+1} \in R_{\sigma}^{-} R_{\sigma}^{-}$; if $0 \leq l-k<t$, then $\left(z_{2 l+1}, z_{2 l+2}\right) \in$ $R_{\sigma}^{-} I^{(t-l+k-1)} \times R_{\sigma}^{-} R_{\sigma}^{-}$. By the last entry in Table 9 , we see that there is some $m$ such that $z_{m}, z_{m+1} \in R_{\sigma}^{-} R_{\sigma}^{-}$. If A5 holds, that is, $0 \leq p<\min \{k, l\}$, then $\left(z_{2 p}, z_{2 p+1}\right) \in J^{(k-p-1)} R_{\sigma}^{-} \times$
TABLE 10

\begin{tabular}{lc}
\hline$\left(z_{-2}, z_{-1}\right) \in I^{(k)} R_{\sigma}^{-} \times I^{(s)} R_{\sigma}^{-}$ & $\left(R_{\sigma}^{-} I^{(s)} \times R_{\sigma}^{-} I^{(k)}\right)$ \\
\hline $0 \leq k \leq s \Rightarrow\left(z_{2 k}, z_{2 k+1}\right) \in R_{\sigma}^{-} R_{\sigma}^{-} \times I^{(s-k-1)} R_{\sigma}^{-}$ & $\left(R_{\sigma}^{-} I^{(s-k-1)} \times R_{\sigma}^{-} R_{\sigma}^{-}\right)$ \\
$0 \leq s<k \Rightarrow\left(z_{2 s}, z_{2 s+1}\right) \in I^{(k-s-1)} R_{\sigma}^{-} \times R_{\sigma}^{-} R_{\sigma}^{-}$ & $\left(R_{\sigma}^{-} R_{\sigma}^{-} \times R_{\sigma}^{-} I^{(k-s-1)}\right)$ \\
\hline
\end{tabular}

TABLE 11

\begin{tabular}{lc}
\hline$\left(z_{-2}, z_{-1}\right) \in J^{(k)} R_{\sigma}^{-} \times R_{\sigma}^{-} J^{(s)}$ & $\left(R_{\sigma}^{-} J^{(k)} \times J^{(s)} R_{\sigma}^{-}\right)$ \\
\hline $0 \leq k \leq s \Rightarrow\left(z_{2 k-1}, z_{2 k}\right) \in R_{\sigma}^{-} J^{(s-k)} \times R_{\sigma}^{-} R_{\sigma}^{-}$ & $\left(J^{(s-k)} R_{\sigma}^{-} \times R_{\sigma}^{-} R_{\sigma}^{-}\right)$ \\
$0 \leq s<k \Rightarrow\left(z_{2 s}, z_{2 s+1}\right) \in J^{(k-s-1)} R_{\sigma}^{-} \times R_{\sigma}^{-} R_{\sigma}^{-}$ & $\left(R_{\sigma}^{-} J^{(k-s-1)} \times R_{\sigma}^{-} R_{\sigma}^{-}\right)$ \\
\hline
\end{tabular}

TABLE 12

\begin{tabular}{lc}
\hline$\left(z_{-2}, z_{-1}\right) \in R_{\sigma}^{-} R_{\sigma}^{-} \times I^{(s)} I^{(k)}$ & $\left(I^{(s)} I^{(k)} \times R_{\sigma}^{-} R_{\sigma}^{-}\right)$ \\
\hline $0 \leq k \leq s \Rightarrow\left(z_{2 k}, z_{2 k+1}\right) \in R_{\sigma}^{-} R_{\sigma}^{-} \times I^{(s-k-1)} R_{\sigma}^{-}$ & $\left(I^{(s-k-1)} R_{\sigma}^{-} \times R_{\sigma}^{-} R_{\sigma}^{-}\right)$ \\
$0 \leq s<k \Rightarrow\left(z_{2 s}, z_{2 s+1}\right) \in R_{\sigma}^{-} R_{\sigma}^{-} \times R_{\sigma}^{-} I^{(k-s-1)}$ & $\left(R_{\sigma}^{-} I^{(k-s-1)} \times R_{\sigma}^{-} R_{\sigma}^{-}\right)$ \\
\hline
\end{tabular}

TABLE 13

\begin{tabular}{ll}
\hline$\left(z_{-2}, z_{-1}\right) \in R_{\sigma}^{-} J^{(k)} \times J^{(p)} I^{(l)}$ & $\left(J^{(k)} R_{\sigma}^{-} \times I^{(l)} J^{(p)}\right)$ \\
\hline $\mathrm{A} 1 \Rightarrow\left(z_{2 k-1}, z_{2 k}\right) \in J^{(p-k)} I^{(l-k)} \times R_{\sigma}^{-} R_{\sigma}^{-}$ & $\left(I^{(l-k)} J^{(p-k)} \times R_{\sigma}^{-} R_{\sigma}^{-}\right)$ \\
$\mathrm{A} 2 \Rightarrow\left(z_{2 k-1}, z_{2 k}\right) \in J^{(p-k)} I^{(l-k)} \times R_{\sigma}^{-} R_{\sigma}^{-}$ & $\left(I^{(l-k)} J^{(p-k)} \times R_{\sigma}^{-} R_{\sigma}^{-}\right)$ \\
$\mathrm{A} 3 \Rightarrow\left(z_{2 l}, z_{2 l+1}\right) \in R_{\sigma}^{-} J^{(k-l-1)} \times R_{\sigma}^{-} R_{\sigma}^{-}$ & $\left(J^{(k-l-1)} R_{\sigma}^{-} \times R_{\sigma}^{-} R_{\sigma}^{-}\right)$ \\
$\mathrm{A} 4 \Rightarrow\left(z_{2 k-1}, z_{2 k}\right) \in J^{(p-k)} I^{(l-k)} \times R_{\sigma}^{-} R_{\sigma}^{-}$ & $\left(I^{(l-k)} J^{(p-k)} \times R_{\sigma}^{-} R_{\sigma}^{-}\right)$ \\
$\mathrm{A} 5 \Rightarrow\left(z_{2 p}, z_{2 p+1}\right) \in R_{\sigma}^{-} J^{(k-p-1)} \times R_{\sigma}^{-} I^{(l-p-1)}\left(J^{(k-p-1)} R_{\sigma}^{-} \times I^{(l-p-1)} R_{\sigma}^{-}\right)$ \\
$\mathrm{A} 6 \Rightarrow\left(z_{2 l}, z_{2 l+1}\right) \in R_{\sigma}^{-} J^{(k-l-1)} \times J^{(p-l-1)} R_{\sigma}^{-}$ & $\left(J^{(k-l-1)} R_{\sigma}^{-} \times R_{\sigma}^{-} J^{(p-l-1)}\right)$ \\
\hline
\end{tabular}

TABLE 14

\begin{tabular}{lc}
\hline$\left(z_{-2}, z_{-1}\right) \in I^{(s)} J^{(p)} \times J^{(k)} R_{\sigma}^{-}$ & $\left(J^{(s)} I^{(p)} \times R_{\sigma}^{-} J^{(k)}\right)$ \\
\hline $0=s<p \Rightarrow\left(z_{-1}, z_{0}\right) \in J^{(k)} R_{\sigma}^{-} \times R_{\sigma}^{-} J^{(p-1)}$ & $\left(R_{\sigma}^{-} J^{(k)} \times R_{\sigma}^{-} I^{(p-1)}\right)$ \\
$0<s<p \Rightarrow\left(z_{-1}, z_{0}\right) \in J^{(k)} R_{\sigma}^{-} \times I^{(s-1)} J^{(p-1)}$ & $\left(R_{\sigma}^{-} J^{(k)} \times J^{(s-1)} I^{(p-1)}\right)$ \\
$0=s=p \Rightarrow\left(z_{-1}, z_{0}\right) \in J^{(k)} R_{\sigma}^{-} \times R_{\sigma}^{-} R_{\sigma}^{-}$ & $\left(R_{\sigma}^{-} J^{(k)} \times R_{\sigma}^{-} R_{\sigma}^{-}\right)$ \\
$0<s=p \Rightarrow\left(z_{-1}, z_{0}\right) \in J^{(k)} R_{\sigma}^{-} \times I^{(s-1)} J^{(p-1)}$ & $\left(R_{\sigma}^{-} J^{(k)} \times J^{(s-1)} I^{(p-1)}\right)$ \\
$0=p<s \Rightarrow\left(z_{-1}, z_{0}\right) \in J^{(k)} R_{\sigma}^{-} \times I^{(s-1)} R_{\sigma}^{-}$ & $\left(R_{\sigma}^{-} J^{(k)} \times J^{(s-1)} R_{\sigma}^{-}\right)$ \\
$0<p<s \Rightarrow\left(z_{-1}, z_{0}\right) \in J^{(k)} R_{\sigma}^{-} \times I^{(s-1)} J^{(p-1)}$ & $\left(R_{\sigma}^{-} J^{(k)} \times J^{(s-1)} I^{(p-1)}\right)$ \\
\hline
\end{tabular}

$I^{(l-p-1)} R_{\sigma}^{-}$. Then, there exists $t \in \mathbf{N}$ such that $\left(z_{2 p}, z_{2 p+1}\right) \in$ $I^{(t)} R_{\sigma}^{-} \times I^{(l-p-1)} R_{\sigma}^{-}$. By Table 10 again, if $0 \leq t \leq l-p-1$, then $\left(z_{2 t+2 p+2}, z_{2 t+2 p+3}\right) \in R_{\sigma}^{-} R_{\sigma}^{-} \times I^{(l-p-1-t-1)} R_{\sigma}^{-}$. By the last entry in Table 9, we see that there is some $m$ such that $z_{m}, z_{m+1} \in$ $R_{\sigma}^{-} R_{\sigma}^{-}$; if $0 \leq l-p-1<t$, then $\left(z_{2 l}, z_{2 l+1}\right) \in R_{\sigma}^{-} I^{(t-l+p)} \times R_{\sigma}^{-} R_{\sigma}^{-}$. By the last entry in Table 9, we see that there is some $m$ such that $z_{m}, z_{m+1} \in R_{\sigma}^{-} R_{\sigma}^{-}$. The other cases are similarly proved.

Consider next the case where $z_{-2} \in R_{\sigma}^{+} R_{\sigma}^{+}$and $z_{-1} \in$ $R_{\sigma}^{+} R_{\sigma}^{-}$. Then, $z_{-2} \in I^{(l)} J^{(p)}$ and $z_{-1} \in J^{(k)} R_{\sigma}^{-}$for some $k, l, p \in$ N. Next, we need to consider six cases: (i) $0=l<p$, (ii) $0<l<p$, (iii) $0=l=p$, (iv) $0<l=p$, (v) $0=p<l$, and (vi) $0<p<l$. Suppose that $0<l<p$ holds, by Table 14, then $\left(z_{-1}, z_{0}\right) \in J^{(k)} R_{\sigma}^{-} \times I^{(l-1)} J^{(p-1)}$. Furthermore, we need to consider six cases: A1-A6. Suppose that A3 holds, that is, $p-1=l-1<k$, then by Table $13,\left(z_{2 l-1}, z_{2 l}\right) \in J^{(k-l)} R_{\sigma}^{-} \times R_{\sigma}^{-} R_{\sigma}^{-}$. Then there exists $t \in \mathbf{N}$ such that $\left(z_{2 l-1}, z_{2 l}\right) \in I^{(t)} R_{\sigma}^{-} \times R_{\sigma}^{-} R_{\sigma}^{-}$. By the third entry in Table 9 , we see that there is some $m$ such that $z_{m}, z_{m+1} \in R_{\sigma}^{-} R_{\sigma}^{-}$. Suppose that A6 holds, that 
is, $l-1<\min \{k, p-1\}$, then by Table $13,\left(z_{2 l-1}, z_{2 l}\right) \in$ $J^{(k-l)} R_{\sigma}^{-} \times R_{\sigma}^{-} J^{(p-l-1)}$. If $k-l \leq p-l-1$, then by Table 11, $\left(z_{2 k}, z_{2 k+1}\right) \in R_{\sigma}^{-} J^{(p-k-l)} \times R_{\sigma}^{-} R_{\sigma}^{-}$. Then, there exists $t \in \mathrm{N}$ such that $\left(z_{2 k}, z_{2 k+1}\right) \in R_{\sigma}^{-} I^{(t)} \times R_{\sigma}^{-} R_{\sigma}^{-}$. By the last entry in Table 9, we see that there is some $m$ such that $z_{m}, z_{m+1} \in R_{\sigma}^{-} R_{\sigma}^{-}$; if $p-l-$ $1<k-l$, then by Table $11,\left(z_{2 p-1}, z_{2 p}\right) \in J^{(k-p)} R_{\sigma}^{-} \times R_{\sigma}^{-} R_{\sigma}^{-}$. Then there exists some $t \in \mathbf{N}$ such that $\left(z_{2 p-1}, z_{2 p}\right) \in I^{(t)} R_{\sigma}^{-} \times R_{\sigma}^{-} R_{\sigma}^{-}$. By the last entry in Table 9, we see that there is some $m$ such that $z_{m}, z_{m+1} \in R_{\sigma}^{-} R_{\sigma}^{-}$. The other cases are similarly proved.

The proof is complete.

Theorem 5. Suppose that $\sigma>1$. Then, every solution of (3) tends to $\overrightarrow{\mathbf{k}}$.

Indeed, let $\left\{z_{n}\right\}_{n=-2}^{\infty}$ be a solution of (3). In view of Lemma 4, one may assume that $z_{-2}, z_{-1} \in R_{\sigma}^{-} R_{\sigma}^{-}$. Then by the $\left(R_{\sigma}^{-} R_{\sigma}^{-}, R_{\sigma}^{-} R_{\sigma}^{-}\right)$entry in Table 7 , one may sees that $z_{0} \in R_{\sigma}^{-} R_{\sigma}^{-}$ and by induction, $z_{n} \in R_{\sigma}^{-} R_{\sigma}^{-}$for all $n \geq-2$. By (14) and (15), then sees that $z_{n} \rightarrow \overrightarrow{\mathbf{k}}$ as required.

\section{The Case $0 \leq \sigma<1$}

Suppose that $0 \leq \sigma<1$. Then,

$$
\begin{gathered}
\sigma>\frac{\sigma-(1-a)}{a}>\frac{\sigma-\left(1-a^{2}\right)}{a^{2}} \\
>\cdots>\frac{\sigma-\left(1-a^{k}\right)}{a^{k}} \longrightarrow-\infty .
\end{gathered}
$$

If we let

$$
\begin{gathered}
K^{(k)}=\left(\frac{\sigma-\left(1-a^{k+1}\right)}{a^{k+1}}, \frac{\sigma-\left(1-a^{k}\right)}{a^{k}}\right], \quad k \in \mathbf{N}, \\
K^{(-1)}=a K^{(0)}+a^{\prime}=(\sigma, a \sigma+(1-a)],
\end{gathered}
$$

then

$$
\begin{gathered}
a K^{(k)}+a^{\prime}=K^{(k-1)}, \quad k \in \mathbf{N}, \\
R_{\sigma}^{+}=\bigcup_{k=0}^{\infty} J^{(k)}, \quad R_{\sigma}^{-}=\bigcup_{k=0}^{\infty} K^{(k)}, \\
R_{\sigma}^{-} R_{\sigma}^{-}=\bigcup_{k=0}^{\infty} K^{(k)} R_{\sigma}^{-}=\bigcup_{k=0}^{\infty} R_{\sigma}^{-} K^{(k)} \\
=\bigcup_{k, s=0}^{\infty} K^{(k)} K^{(s)}, \\
R_{\sigma}^{+} R_{\sigma}^{+}=\bigcup_{k=0}^{\infty} J^{(k)} R_{\sigma}^{+}=\bigcup_{k=0}^{\infty} R_{\sigma}^{+} J^{(k)} \\
=\bigcup_{k, s=0}^{\infty} J^{(k)} J^{(s)},
\end{gathered}
$$

$$
\begin{aligned}
R_{\sigma}^{+} R_{\sigma}^{-} & =\bigcup_{k=0}^{\infty} J^{(k)} R_{\sigma}^{-}=\bigcup_{k=0}^{\infty} R_{\sigma}^{+} K^{(k)} \\
& =\bigcup_{k, s=0}^{\infty} J^{(k)} K^{(s)}, \\
R_{\sigma}^{-} R_{\sigma}^{+} & =\bigcup_{k=0}^{\infty} K^{(k)} R_{\sigma}^{+}=\bigcup_{k=0}^{\infty} R_{\sigma}^{-} J^{(k)} \\
& =\bigcup_{k, s=0}^{\infty} K^{(k)} J^{(s)} .
\end{aligned}
$$

Let $\left\{z_{n}\right\}_{n=-2}^{\infty}$ be a solution of (3). Let us consider first the case where $z_{n}, z_{n+1} \in R_{\sigma}^{+} R_{\sigma}^{-}$. Then by (7) and (16), we see that

$$
z_{n+2}=a z_{n}+a^{\prime} \overrightarrow{\mathbf{i}}
$$

Note that (in view of our assumption that $0 \leq \sigma<1$ )

$$
\begin{aligned}
a R_{\sigma}^{+} R_{\sigma}^{-}+a^{\prime} F_{\sigma}\left(R_{\sigma}^{+} R_{\sigma}^{-}\right)= & a R_{\sigma}^{+} R_{\sigma}^{-}+a^{\prime} \overrightarrow{\mathbf{i}} \\
= & a((\sigma, \infty) \times(-\infty, \sigma]) \\
& +(1-a)(1,-1) \\
= & (a \sigma+(1-a), \infty) \\
& \times(-\infty, a \sigma-(1-a)] \\
\subseteq & R_{\sigma}^{+} R_{\sigma}^{-} .
\end{aligned}
$$

Hence, we may see further that $z_{n+2} \in R_{\sigma}^{+} R_{\sigma}^{-}$. Similarly, since

$$
\begin{aligned}
a R_{\sigma}^{+} R_{\sigma}^{+}+a^{\prime} F_{\sigma}\left(R_{\sigma}^{-} R_{\sigma}^{-}\right)= & a R_{\sigma}^{+} R_{\sigma}^{+}+a^{\prime} \overrightarrow{\mathbf{k}} \\
= & a((\sigma, \infty) \times(\sigma, \infty)) \\
& +(1-a)(1,1) \\
= & (a \sigma+(1-a), \infty) \\
& \times(a \sigma+(1-a), \infty) \\
\subseteq & R_{\sigma}^{+} R_{\sigma}^{+}, \\
a R_{\sigma}^{-} R_{\sigma}^{-}+a^{\prime} F_{\sigma}\left(R_{\sigma}^{+} R_{\sigma}^{+}\right)= & a R_{\sigma}^{-} R_{\sigma}^{-}-a^{\prime} \overrightarrow{\mathbf{k}} \\
= & a((-\infty, \sigma] \times(-\infty, \sigma]) \\
& -(1-a)(1,1) \\
= & (-\infty, a \sigma-(1-a)] \\
& \times(-\infty, a \sigma-(1-a)] \\
\subseteq & R_{\sigma}^{-} R_{\sigma}^{-},
\end{aligned}
$$

we see that if $\left(z_{n}, z_{n+1}\right) \in R_{\sigma}^{+} R_{\sigma}^{+} \times R_{\sigma}^{-} R_{\sigma}^{-}$, then $z_{n+2} \in R_{\sigma}^{+} R_{\sigma}^{+}$ and $z_{n+3} \in R_{\sigma}^{-} R_{\sigma}^{-}$. By similar considerations, we may build a table (consisting of two separate parts Tables 15 and 16) which plays the same role as Table 1 . 
TABLE 15

\begin{tabular}{lccccc}
\hline & $K^{(s)} R_{\sigma}^{-}$ & $R_{\sigma}^{-} K^{(s)}$ & $K^{(s)} K^{(l)}$ & $J^{(s)} R_{\sigma}^{-}$ & $R_{\sigma}^{+} K^{(l)}$ \\
\hline$K^{(k)} R_{\sigma}^{-}$ & & & $K^{(k-1)} R_{\sigma}^{-}$ & $K^{(k-1)} R_{\sigma}^{-}$ \\
$R_{\sigma}^{-} K^{(k)}$ & & & & $K^{(k-1)} R_{\sigma}^{-}$ \\
$K^{(k)} K^{(p)}$ & $K^{(k-1)} K^{(p-1)}$ & $K^{(k-1)} K^{(p-1)}$ & $K^{(k-1)} K^{(p-1)}$ & & $R_{\sigma}^{+} R_{\sigma}^{-}$ \\
$J^{(k)} R_{\sigma}^{-}$ & & & $R_{\sigma}^{+} R_{\sigma}^{-}$ & $R_{\sigma}^{+} R_{\sigma}^{-}$ \\
$J^{(k)} K^{(s)}$ & & & $R_{\sigma}^{+} R_{\sigma}^{-}$ & $R_{\sigma}^{+} R_{\sigma}^{-}$ \\
$R_{\sigma}^{+} K^{(k)}$ & $R_{\sigma}^{+} K^{(k-1)}$ & $R_{\sigma}^{+} K^{(k-1)}$ & $R_{\sigma}^{+} K^{(k-1)}$ & $R_{\sigma}^{+} R_{\sigma}^{-}$ & $R_{\sigma}^{+} R^{-}$ \\
$R_{\sigma}^{-} K^{(k)}$ & & & & $K^{(k-1)} J^{(p-1)}$ \\
$K^{(k)} R_{\sigma}^{+}$ & $K^{(k-1)} R_{\sigma}^{+}$ & $K^{(k-1)} R_{\sigma}^{+}$ & $K^{(k-1)} R_{\sigma}^{+}$ & & $K_{\sigma}^{(k-1)} J^{(p-1)}$ \\
$K^{(k)} J^{(p)}$ & & & & $K^{(k-1)} J^{(p-1)}$ & $R_{\sigma}^{+} J^{(k-1)}$ \\
$R_{\sigma}^{+} J^{(k)}$ & $R_{\sigma}^{+} R_{\sigma}^{+}$ & $R_{\sigma}^{+} R_{\sigma}^{+}$ & & $R_{\sigma}^{+} J^{(k-1)}$ \\
$J^{(k)} R_{\sigma}^{+}$ & $R_{\sigma}^{+} R_{\sigma}^{+}$ & $R_{\sigma}^{+} R_{\sigma}^{+}$ & $R_{\sigma}^{+} R_{\sigma}^{+}$ & & \\
$J^{(k)} J^{(p)}$ & $R_{\sigma}^{+} R_{\sigma}^{+}$ & $R_{\sigma}^{+} R_{\sigma}^{+}$ & $R_{\sigma}^{+} R_{\sigma}^{+}$ & &
\end{tabular}

Table 16

\begin{tabular}{lccccc}
\hline & $R_{\sigma}^{-} J^{(s)}$ & $K^{(s)} R_{\sigma}^{+}$ & $K^{(s)} J^{(l)}$ & $R_{\sigma}^{+} J^{(s)}$ & $J^{(s)} R_{\sigma}^{+}$ \\
\hline$K^{(k)} R_{\sigma}^{-}$ & & & $R_{\sigma}^{-} R_{\sigma}^{-}$ & $R_{\sigma}^{-} R_{\sigma}^{-}$ \\
$R_{\sigma}^{-} K^{(k)}$ & & $R_{\sigma}^{-} K^{(k-1)}$ & $R_{\sigma}^{-} K^{(k-1)}$ & $R_{\sigma}^{-} R_{\sigma}^{-}$ & $R_{\sigma}^{-} R_{\sigma}^{-}$ \\
$K^{(k)} K^{(p)}$ & $R_{\sigma}^{-} K^{(k-1)}$ & & $R_{\sigma}^{-} R_{\sigma}^{-}$ & $R_{\sigma}^{-} R_{\sigma}^{-}$ \\
$J^{(k)} R_{\sigma}^{-}$ & & & $J^{-}$ & $J^{-} R_{\sigma}^{-}$ \\
$J^{(k)} K^{(s)}$ & $J^{(k-1)} R_{\sigma}^{-}$ & & $J_{\sigma}^{-}$ \\
$R_{\sigma}^{+} K^{(k)}$ & & & & $R_{\sigma}^{-} J^{(k-1)} R_{\sigma}^{-}$ \\
$R_{\sigma}^{-} J^{(k)}$ & $R_{\sigma}^{-} R_{\sigma}^{+}$ & $J^{(k-1)} K^{(s-1)}$ & & $R_{\sigma}^{-} J^{(k-1)}$ & \\
$K^{(k)} R_{\sigma}^{+}$ & $R_{\sigma}^{-} R_{\sigma}^{+}$ & $R_{\sigma}^{-} R_{\sigma}^{+}$ & $R_{\sigma}^{-} R_{\sigma}^{+}$ & \\
$K^{(k)} J^{(p)}$ & $R_{\sigma}^{-} R_{\sigma}^{+}$ & $R_{\sigma}^{-} R_{\sigma}^{+}$ & $R_{\sigma}^{-} R_{\sigma}^{+}$ & & $R_{\sigma}^{-} J^{(k-1)}$ \\
$R_{\sigma}^{+} J^{(k)}$ & $R_{\sigma}^{-} R_{\sigma}^{+}$ & $R_{\sigma}^{-} R_{\sigma}^{+}$ & & \\
$J^{(k)} R_{\sigma}^{+}$ & $J^{(k-1)} R_{\sigma}^{+}$ & $J^{(k-1)} R_{\sigma}^{+}$ & $J^{(k-1)} R_{\sigma}^{+}$ & & $J^{(k-1)} J^{(p-1)}$ \\
$J^{(k)} J^{(p)}$ & & & & $J^{(k-1)} J^{(p-1)}$ & $J^{(k-1)} J^{(p-1)}$ \\
\hline
\end{tabular}

TABle 17

\begin{tabular}{ccccc}
\hline & $R_{\sigma}^{-} R_{\sigma}^{-}$ & $R_{\sigma}^{+} R_{\sigma}^{-}$ & $R_{\sigma}^{-} R_{\sigma}^{+}$ & $R_{\sigma}^{+} R_{\sigma}^{+}$ \\
\hline$R_{\sigma}^{-} R_{\sigma}^{-}$ & See Table 8 & See Table 18 & See Table 18 & $\langle-\overrightarrow{\mathbf{k}}, \overrightarrow{\mathbf{k}}\rangle$ \\
$R_{\sigma}^{+} R_{\sigma}^{-}$ & See Table 5 & $\langle\overrightarrow{\mathbf{i}}\rangle$ & See Table 7 & See Table 6 \\
$R_{\sigma}^{-} R_{\sigma}^{+}$ & See Table 5 & See Table 7 & $\langle-\overrightarrow{\mathbf{i}}\rangle$ & See Table 6 \\
$R_{\sigma}^{+} R_{\sigma}^{+}$ & $\langle\overrightarrow{\mathbf{k}},-\overrightarrow{\mathbf{k}}\rangle$ & See Table 4 & See Table 4 & See Table 8 \\
\hline
\end{tabular}

TABle 18

\begin{tabular}{lc}
\hline$\left(z_{-2}, z_{-1}\right) \in K^{(k)} R_{\sigma}^{-} \times R_{\sigma}^{+} K^{(s)}$ & $\left(R_{\sigma}^{-} K^{(k)} \times K^{(s)} R_{\sigma}^{+}\right)$ \\
\hline $0 \leq k \leq s \Rightarrow\left(z_{2 k}, z_{2 k+1}\right) \in R_{\sigma}^{+} R_{\sigma}^{-} \times R_{\sigma}^{+} R_{\sigma}^{-}$ & $\left(R_{\sigma}^{-} R_{\sigma}^{+} \times R_{\sigma}^{-} R_{\sigma}^{+}\right)$ \\
$0 \leq s<k \Rightarrow\left(z_{2 s}, z_{2 s+1}\right) \in R_{\sigma}^{-} R_{\sigma}^{-} \times R_{\sigma}^{+} R_{\sigma}^{+}$ & $\left(R_{\sigma}^{-} R_{\sigma}^{-} \times R_{\sigma}^{+} R_{\sigma}^{+}\right)$ \\
\hline
\end{tabular}

TAble 19

\begin{tabular}{ll}
\hline$\left(z_{-2}, z_{-1}\right) \in J^{(k)} R_{\sigma}^{+} \times R_{\sigma}^{-} J^{(s)}$ & $\left(R_{\sigma}^{+} J^{(k)} \times J^{(s)} R_{\sigma}^{-}\right)$ \\
\hline $0 \leq k \leq s \Rightarrow\left(z_{2 k}, z_{2 k+1}\right) \in R_{\sigma}^{-} R_{\sigma}^{+} \times R_{\sigma}^{-} R_{\sigma}^{+}$ & $\left(R_{\sigma}^{+} R_{\sigma}^{-} \times R_{\sigma}^{+} R_{\sigma}^{-}\right)$ \\
$0 \leq s<k \Rightarrow\left(z_{2 s}, z_{2 s+1}\right) \in R_{\sigma}^{+} R_{\sigma}^{+} \times R_{\sigma}^{-} R_{\sigma}^{-}$ & $\left(R_{\sigma}^{+} R_{\sigma}^{+} \times R_{\sigma}^{-} R_{\sigma}^{-}\right)$ \\
\hline
\end{tabular}

Next, we let $\left\{z_{n}\right\}_{n=-2}^{\infty}$ be a solution of (3) again. If $\left(z_{-2}, z_{-1}\right) \in R_{\sigma}^{+} R_{\sigma}^{+} \times R_{\sigma}^{-} R_{\sigma}^{-}$, then by the $\left(R_{\sigma}^{+} J^{(k)}, R_{\sigma}^{-} I^{(s)}\right)$ entry of Table 15 , we see that $z_{0} \in R_{\sigma}^{+} R_{\sigma}^{+}$. Now that $\left(z_{-1}, z_{0}\right) \in$
TABle 20

\begin{tabular}{lc}
\hline$\left(z_{-2}, z_{-1}\right) \in R_{\sigma}^{+} K^{(k)} \times K^{(s)} R_{\sigma}^{-}$ & $\left(K^{(k)} R_{\sigma}^{+} \times R_{\sigma}^{-} K^{(s)}\right)$ \\
\hline $0 \leq k \leq s \Rightarrow\left(z_{2 k}, z_{2 k+1}\right) \in R_{\sigma}^{+} R_{\sigma}^{+} \times R_{\sigma}^{-} R_{\sigma}^{-}$ & $\left(R_{\sigma}^{+} R_{\sigma}^{+} \times R_{\sigma}^{-} R_{\sigma}^{-}\right)$ \\
$0 \leq s<k \Rightarrow\left(z_{2 s}, z_{2 s+1}\right) \in R_{\sigma}^{+} R_{\sigma}^{-} \times R_{\sigma}^{+} R_{\sigma}^{-}$ & $\left(R_{\sigma}^{-} R_{\sigma}^{+} \times R_{\sigma}^{-} R_{\sigma}^{+}\right)$ \\
\hline & \\
TABLE 21 & \\
\hline \multicolumn{2}{c}{} \\
\hline $0 \leq k \leq s \Rightarrow\left(z_{2 k}, z_{2 k+1}\right) \in R_{\sigma}^{-} R_{\sigma}^{-} \times R_{\sigma}^{+} R_{\sigma}^{+}$ & $\left(J^{(k)} R_{\sigma}^{-} \times R_{\sigma}^{+} J^{(s)}\right)$ \\
$0 \leq s<k \Rightarrow\left(z_{2 s}, z_{2 s+1}\right) \in R_{\sigma}^{-} R_{\sigma}^{+} \times R_{\sigma}^{-} R_{\sigma}^{+}$ & $\left(R_{\sigma}^{+} R_{\sigma}^{-} \times R_{\sigma}^{+} R_{\sigma}^{+}\right)$ \\
\hline
\end{tabular}

$R_{\sigma}^{-} R_{\sigma}^{-} \times R_{\sigma}^{+} R_{\sigma}^{+}$, we may use the $\left(K^{(k)} R_{\sigma}^{-}, J^{(s)} R_{\sigma}^{+}\right)$entry of Table 16 to infer $z_{1} \in R_{\sigma}^{-} R_{\sigma}^{-}$again. By induction, we see that $z_{2 n} \in$ $R_{\sigma}^{+} R_{\sigma}^{+}, z_{2 n+1} \in R_{\sigma}^{-} R_{\sigma}^{-}$for all $n \geq-2$. By (7), we then see that

$$
\begin{gathered}
z_{2 n}=a z_{n-2}+a^{\prime} \overrightarrow{\mathbf{k}}, \quad n \in \mathbf{N}, \\
z_{2 n+1}=a z_{2 n-1}-a^{\prime} \overrightarrow{\mathbf{k}}, \quad n \in \mathbf{N},
\end{gathered}
$$

and hence by (14) and (15), we see that $z_{2 n} \rightarrow \overrightarrow{\mathbf{k}}, z_{2 n+1} \rightarrow-\overrightarrow{\mathbf{k}}$ as $n \rightarrow \infty$. By considering $z_{-2}$ and $z_{-1}$ in different parts of the plane, we may apply the same principle to obtain Table 17.

In this table, there are twelve indeterminate cases. Let us go through one case. Let $\left(z_{-2}, z_{-1}\right) \in R_{\sigma}^{-} R_{\sigma}^{-} \times R_{\sigma}^{+} R_{\sigma}^{-}$. Then, 
TABLE 22

\begin{tabular}{lc}
\hline$\left(z_{-2}, z_{-1}\right) \in K^{(k)} J^{(p)} \times J^{(l)} K^{(m)}$ & $\left(J^{(k)} K^{(p)} \times K^{(l)} J^{(m)}\right)$ \\
\hline $\mathrm{B} 1 \Rightarrow\left(z_{2 k}, z_{2 k+1}\right) \in R_{\sigma}^{+} R_{\sigma}^{-} \times R_{\sigma}^{+} R_{\sigma}^{-}$ & $\left(R_{\sigma}^{-} R_{\sigma}^{+} \times R_{\sigma}^{-} R_{\sigma}^{+}\right)$ \\
B2 $\Rightarrow\left(z_{2 k}, z_{2 k+1}\right) \in R_{\sigma}^{+} J^{(p-k-1)} \times J^{(l-k-1)} R_{\sigma}^{-}$ & $\left(R_{\sigma}^{-} K^{(p-k-1)} \times K^{(l-k-1)} R_{\sigma}^{+}\right)$ \\
B3 $\Rightarrow\left(z_{2 p}, z_{2 p+1}\right) \in K^{(k-p-1)} R_{\sigma}^{-} \times R_{\sigma}^{+} K^{(m-p-1)}$ & $\left(J^{(k-p-1)} R_{\sigma}^{+} \times R_{\sigma}^{-} J^{(m-p-1)}\right)$ \\
B4 $\Rightarrow\left(z_{2 p}, z_{2 p+1}\right) \in K^{(k-p-1)} R_{\sigma}^{-} \times R_{\sigma}^{+} K^{(m-p-1)}$ & $\left(J^{(k-p-1)} R_{\sigma}^{+} \times R_{\sigma}^{-} J^{(m-p-1)}\right)$ \\
B5 $\Rightarrow\left(z_{2 p}, z_{2 p+1}\right) \in R_{\sigma}^{-} R_{\sigma}^{-} \times R_{\sigma}^{+} R_{\sigma}^{+}$ & $\left(R_{\sigma}^{+} R_{\sigma}^{+} \times R_{\sigma}^{-} R_{\sigma}^{-}\right)$ \\
B6 $\Rightarrow\left(z_{2 l}, z_{2 l+1}\right) \in K^{(k-l-1)} R_{\sigma}^{+} \times R_{\sigma}^{-} K^{(m-l-1)}$ & $\left(J^{(k-l-1)} R_{\sigma}^{-} \times R_{\sigma}^{+} J^{(m-l-1)}\right)$ \\
B7 $\Rightarrow\left(z_{2 l}, z_{2 l+1}\right) \in R_{\sigma}^{-} R_{\sigma}^{+} \times R_{\sigma}^{-} R_{\sigma}^{+}$ & $\left(R_{\sigma}^{+} R_{\sigma}^{-} \times R_{\sigma}^{+} R_{\sigma}^{-}\right)$ \\
B8 $\Rightarrow\left(z_{2 l}, z_{2 l+1}\right) \in R_{\sigma}^{+} R_{\sigma}^{+} \times R_{\sigma}^{-} R_{\sigma}^{-}$ & $\left(R_{\sigma}^{-} R_{\sigma}^{-} \times R_{\sigma}^{+} R_{\sigma}^{+}\right)$ \\
B9 $\Rightarrow\left(z_{2 m}, z_{2 m+1}\right) \in R_{\sigma}^{-} J^{(p-m-1)} \times J^{(l-m-1)} R_{\sigma}^{+}$ & $\left(R_{\sigma}^{+} K^{(p-m-1)} \times K^{(l-m-1)} R_{\sigma}^{-}\right)$ \\
B10 $\Rightarrow\left(z_{2 m}, z_{2 m+1}\right) \in R_{\sigma}^{+} J^{(p-m-1)} \times J^{(l-m-1)} R_{\sigma}^{-}$ & $\left(R_{\sigma}^{-} K^{(p-m-1)} \times K^{(l-m-1)} R_{\sigma}^{+}\right)$ \\
B11 $\Rightarrow\left(z_{2 p}, z_{2 p+1}\right) \in R_{\sigma}^{-} R_{\sigma}^{-} \times R_{\sigma}^{+} R_{\sigma}^{+}$ & $\left(R_{\sigma}^{+} R_{\sigma}^{+} \times R_{\sigma}^{-} R_{\sigma}^{-}\right)$ \\
B12 $\Rightarrow\left(z_{2 l}, z_{2 l+1}\right) \in R_{\sigma}^{+} R_{\sigma}^{+} \times R_{\sigma}^{-} R_{\sigma}^{-}$ & $\left(R_{\sigma}^{-} R_{\sigma}^{-} \times R_{\sigma}^{+} R_{\sigma}^{+}\right)$ \\
\hline
\end{tabular}

TABLE 23

\begin{tabular}{lc}
\hline$\left(z_{-2}, z_{-1}\right) \in J^{(k)} J^{(p)} \times J^{(l)} J^{(m)}$ & $\left(K^{(k)} K^{(p)} \times K^{(l)} K^{(m)}\right)$ \\
\hline $\mathrm{B} 1 \Rightarrow\left(z_{2 k}, z_{2 k+1}\right) \in R_{\sigma}^{-} R_{\sigma}^{-} \times R_{\sigma}^{+} R_{\sigma}^{+}$ & $\left(R_{\sigma}^{+} R_{\sigma}^{+} \times R_{\sigma}^{-} R_{\sigma}^{-}\right)$ \\
$\mathrm{B} 2 \Rightarrow\left(z_{2 k}, z_{2 k+1}\right) \in R_{\sigma}^{-} J^{(p-k-1)} \times J^{(l-k-1)} R_{\sigma}^{+}$ & $\left(R_{\sigma}^{+} K^{(p-k-1)} \times K^{(l-k-1)} R_{\sigma}^{-}\right)$ \\
$\mathrm{B} 3 \Rightarrow\left(z_{2 p}, z_{2 p+1}\right) \in J^{(k-p-1)} R_{\sigma}^{-} \times R_{\sigma}^{+} J^{(m-p-1)}$ & $\left(K^{(k-p-1)} R_{\sigma}^{+} \times R_{\sigma}^{-} K^{(m-p-1)}\right)$ \\
$\mathrm{B} 4 \Rightarrow\left(z_{2 p}, z_{2 p+1}\right) \in J^{(k-p-1)} R_{\sigma}^{-} \times R_{\sigma}^{+} J^{(m-p-1)}$ & $\left(K^{(k-p-1)} R_{\sigma}^{+} \times R_{\sigma}^{-} K^{(m-p-1)}\right)$ \\
$\mathrm{B} 5 \Rightarrow\left(z_{2 p}, z_{2 p+1}\right) \in R_{\sigma}^{+} R_{\sigma}^{-} \times R_{\sigma}^{+} R_{\sigma}^{-}$ & $\left(R_{\sigma}^{-} R_{\sigma}^{+} \times R_{\sigma}^{-} R_{\sigma}^{+}\right)$ \\
$\mathrm{B} 6 \Rightarrow\left(z_{2 l}, z_{2 l+1}\right) \in J^{(k-l-1)} R_{\sigma}^{+} \times R_{\sigma}^{-} J^{(m-l-1)}$ & $\left(K^{(k-l-1)} R_{\sigma}^{-} \times R_{\sigma}^{+} K^{(m-l-1)}\right)$ \\
$\mathrm{B} 7 \Rightarrow\left(z_{2 l}, z_{2 l+1}\right) \in R_{\sigma}^{+} R_{\sigma}^{+} \times R_{\sigma}^{-} R_{\sigma}^{-}$ & $\left(R_{\sigma}^{-} R_{\sigma}^{-} \times R_{\sigma}^{+} R_{\sigma}^{+}\right)$ \\
$\mathrm{B} 8 \Rightarrow\left(z_{2 l}, z_{2 l+1}\right) \in R_{\sigma}^{-} R_{\sigma}^{+} \times R_{\sigma}^{-} R_{\sigma}^{+}$ & $\left(R_{\sigma}^{+} R_{\sigma}^{-} \times R_{\sigma}^{+} R_{\sigma}^{-}\right)$ \\
$\mathrm{B} 9 \Rightarrow\left(z_{2 m}, z_{2 m+1}\right) \in R_{\sigma}^{+} J^{(p-m-1)} \times J^{(l-m-1)} R_{\sigma}^{-}$ & $\left(R_{\sigma}^{-} K^{(p-m-1)} \times K^{(l-m-1)} R_{\sigma}^{+}\right)$ \\
$\mathrm{B} 10 \Rightarrow\left(z_{2 m}, z_{2 m+1}\right) \in R_{\sigma}^{-} J^{(p-m-1)} \times J^{(l-m-1)} R_{\sigma}^{+}$ & $\left(R_{\sigma}^{+} K^{(p-m-1)} \times K^{(l-m-1)} R_{\sigma}^{-}\right)$ \\
B11 $\Rightarrow\left(z_{2 p}, z_{2 p+1}\right) \in R_{\sigma}^{+} R_{\sigma}^{-} \times R_{\sigma}^{+} R_{\sigma}^{-}$ & $\left(R_{\sigma}^{-} R_{\sigma}^{+} \times R_{\sigma}^{-} R_{\sigma}^{+}\right)$ \\
B12 $\Rightarrow\left(z_{2 l}, z_{2 l+1}\right) \in R_{\sigma}^{-} R_{\sigma}^{+} \times R_{\sigma}^{-} R_{\sigma}^{+}$ & $\left(R_{\sigma}^{+} R_{\sigma}^{-} \times R_{\sigma}^{+} R_{\sigma}^{-}\right)$ \\
\hline
\end{tabular}

$\left(z_{-2}, z_{-1}\right) \in K^{(k)} R_{\sigma}^{-} \times R_{\sigma}^{+} K^{(s)}$ for some $k, s \in \mathbf{N}$. There are then two subcases: (i) $0 \leq k \leq s$ or (ii) $0 \leq s<k$. In the former case, by Table 15 ,

$$
z_{0}=a z_{-2}+a^{\prime} F_{\sigma}\left(z_{-1}\right) \in K^{(k-1)} R_{\sigma}^{-},
$$

and (hence)

$$
z_{1}=a z_{-1}+a^{\prime} F_{\sigma}\left(z_{0}\right) \in R_{\sigma}^{+} K^{(s-1)},
$$

and so forth, and finally,

$$
\begin{gathered}
z_{2 k} \in K^{(-1)} R_{\sigma}^{-} \subseteq R_{\sigma}^{+} R_{\sigma}^{-}, \\
z_{2 k+1} \in R_{\sigma}^{+} R_{\sigma}^{-} .
\end{gathered}
$$

In the latter case, we may similarly show that $\left(z_{2 s}, z_{2 s+1}\right) \in$ $R_{\sigma}^{-} R_{\sigma}^{-} \times R_{\sigma}^{+} R_{\sigma}^{+}$. By considering $\left(z_{-2}, z_{-1}\right)$ in different parts, we may then construct four self-explanatory Tables 18, 19, 20, and 21.

As a consequence, if $\left(z_{-2}, z_{-1}\right) \in K^{(k)} R^{-} \times R^{+} K^{(s)}$ and $0 \leq$ $k \leq s$, then the solution of (3) originated from $z_{-2}$ and $z_{-1}$ will satisfy $\left(z_{2 k}, z_{2 k+1}\right) \in R_{\sigma}^{+} R_{\sigma}^{-} \times R_{\sigma}^{+} R_{\sigma}^{-}$. Then by Table 17, $z_{n} \rightarrow$ $\langle\vec{i}\rangle$.

Next, let $\left(z_{-2}, z_{-1}\right) \in R_{\sigma}^{-} R_{\sigma}^{+} \times R_{\sigma}^{+} R_{\sigma}^{-}$. Then, $\left(z_{-2}, z_{-1}\right) \in$ $K^{(k)} J^{(p)} \times J^{(l)} K^{(m)}$ for some $k, p, l, m \in \mathbf{N}$. As mentioned before, there are twelve different ordering arrangements B1$\mathrm{B} 12$ for the nonnegative integers $k, p, l$, and $m$. In case B2 holds, we may make use of Table 22 repeatedly to show that $\left(z_{2 k}, z_{2 k+1}\right) \in R_{\sigma}^{+} J^{(p-k-1)} \times J^{(l-k-1)} R_{\sigma}^{-}$(which is recorded in self-explanatory Tables 22 and 23). If $0 \leq p-k-1 \leq l-k-1$, then by Table $19,\left(z_{2 p}, z_{2 p+1}\right) \in R_{\sigma}^{+} R_{\sigma}^{-} \times R_{\sigma}^{+} R_{\sigma}^{-}$. By Table 17 again, $z_{n} \rightarrow\langle\overrightarrow{\mathbf{i}}\rangle$.

In conclusion, we have shown the following result.

Theorem 6. Suppose that $0 \leq \sigma<1$. Then, a solution of (3) must be either asymptotically 1-periodic tending to the limit cycles $\langle\overrightarrow{\mathbf{i}}\rangle$ or $\langle-\overrightarrow{\mathbf{i}}\rangle$ or asymptotically 2-periodic tending to the limit cycle $\langle\overrightarrow{\mathbf{k}},-\overrightarrow{\mathbf{k}}\rangle$.

We remark that, as can be seen from the above exhaustive arguments and Example 2, the region of attraction of each 
limit cycle can be given precisely (by implementing a simple computer program, if necessary).

\section{Conclusions}

In neural network terminologies, we have discussed a simple two-neuron recurrent McCulloch-Pitts-type neural network with a nonnegative threshold $\sigma$. For $\sigma>1$, all solutions tend to the limiting state $(1,1)$; for $\sigma=1$, all solutions must either be tending to the limiting states $(-1,1),(1,-1)$ or $(1,1)$, or asymptotically 2 -periodic tending to the limit cycles $\langle(1,-1),(1,1)\rangle,\langle(-1,1),(1,1)\rangle$, or $\langle(-1,-1),(1,1)\rangle$; and for $0 \leq \sigma<1$, all solutions must either be tending to the steady states $(-1,1)$, or $(1,-1)$ or asymptotically 2 -periodic tending to the limit cycle $\langle(1,1),(-1,-1)\rangle$. In all cases, the corresponding region of attraction of each limit cycle can be given.

Our proofs show that more general multiple-neuron recurrent McCulloch-Pitts-type neural networks possess similar behaviors. However, the derivations may involve more delicate graph theoretic arguments and are better left for other studies.

\section{References}

[1] C. Hou and S. S. Cheng, "Eventually periodic solutions for difference equations with periodic coefficients and nonlinear control functions," Discrete Dynamics in Nature and Society, vol. 2008, Article ID 179589, 21 pages, 2008.

[2] Q. Ge, C. Hou, and S. S. Cheng, "Complete asymptotic analysis of a nonlinear recurrence relation with threshold control," Advances in Difference Equations, vol. 2010, Article ID 143849, 19 pages, 2010.

[3] W. S. McCulloch and W. Pitts, "A logical calculus of the ideas immanent in nervous activity, Bulletin of Mathematical Biophysics, vol. 5, pp. 115-133, 1943. 


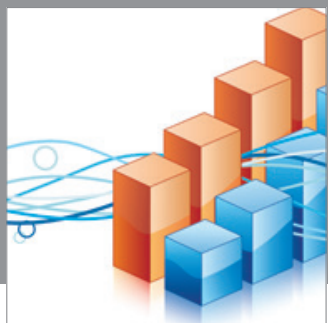

Advances in

Operations Research

mansans

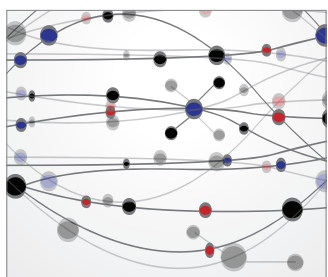

The Scientific World Journal
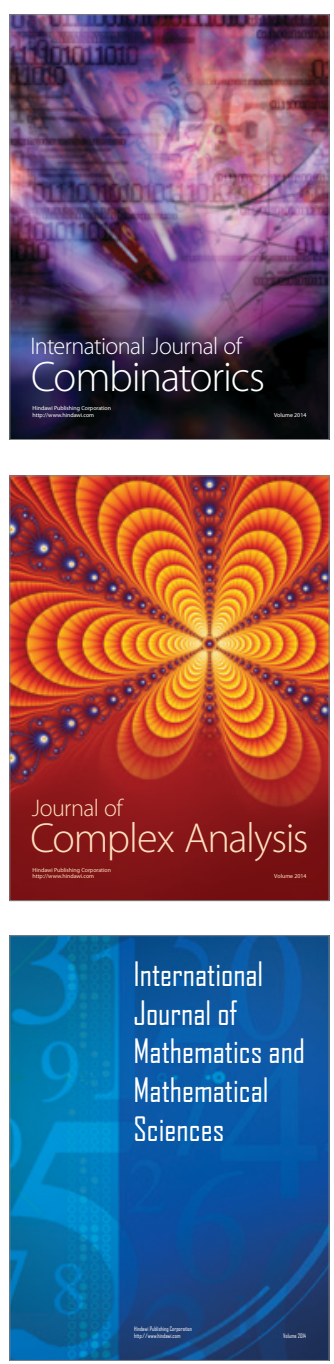
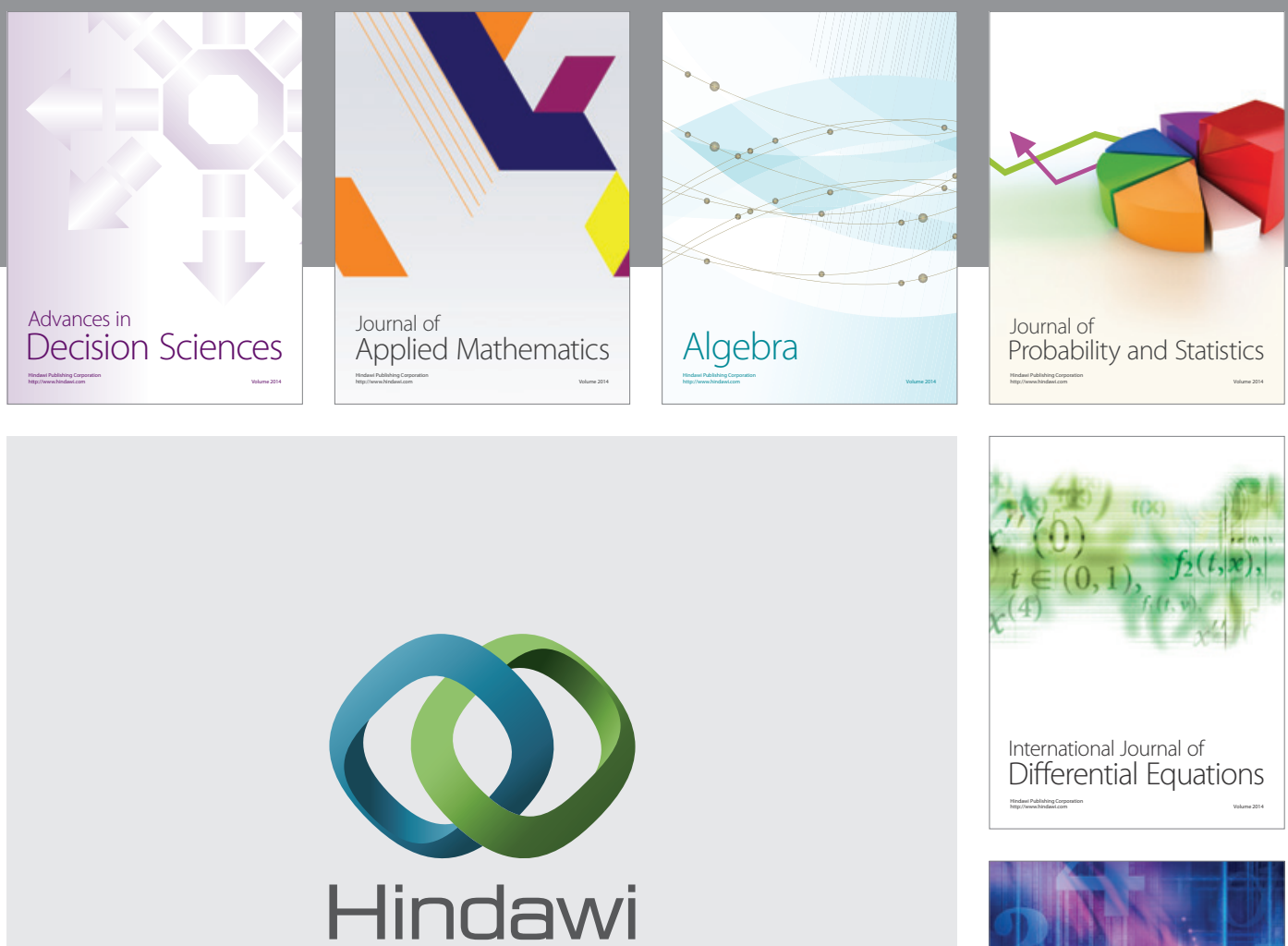

Submit your manuscripts at http://www.hindawi.com
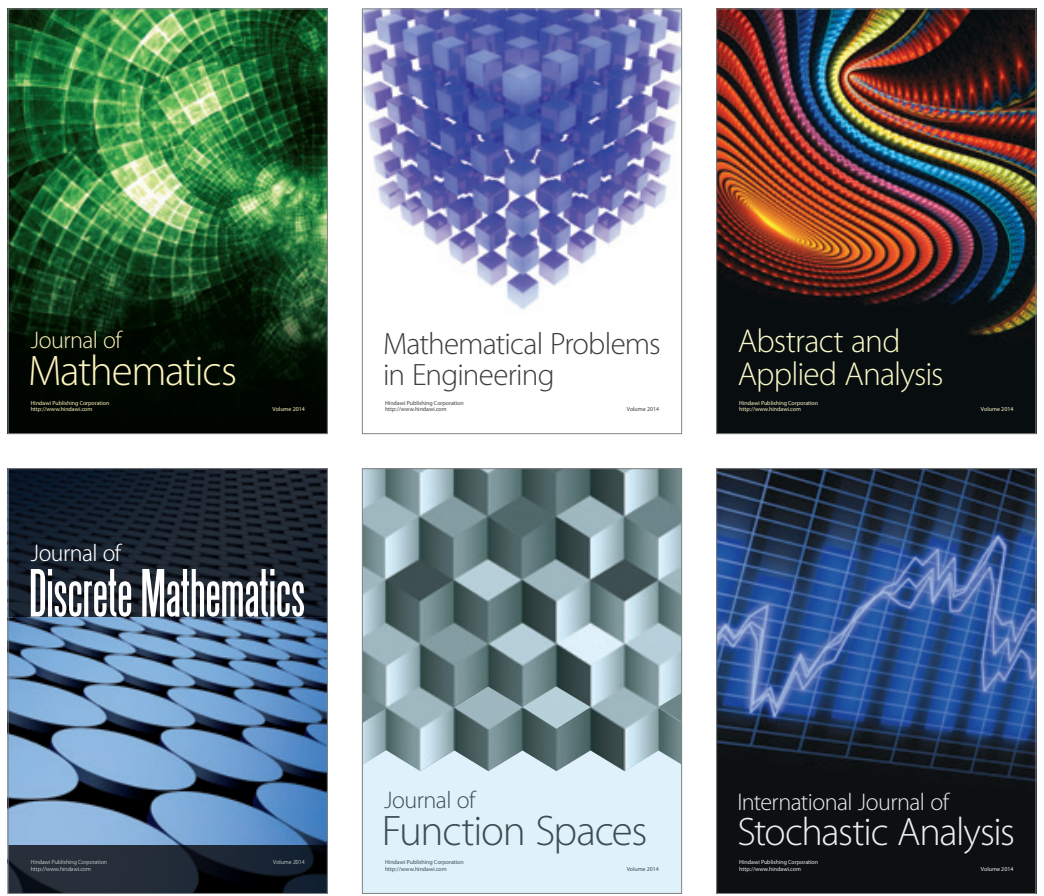

Journal of

Function Spaces

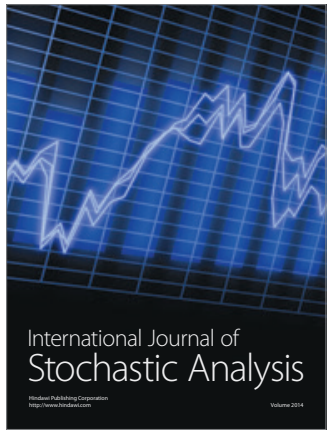

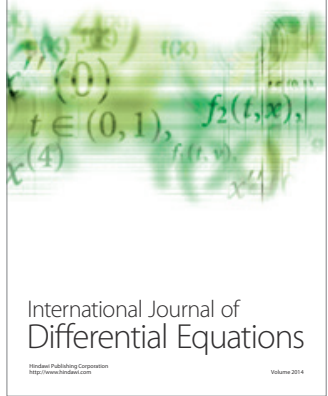
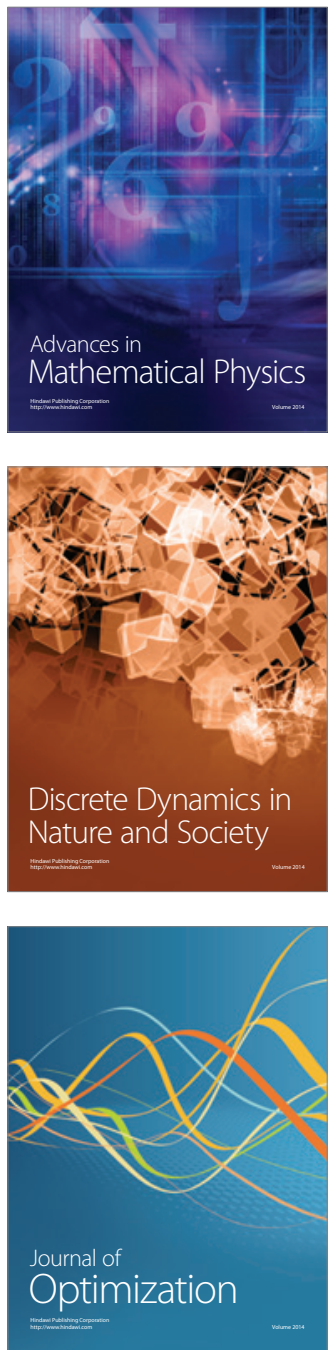